\title{
FGFR4 phosphorylates MST1 to confer breast cancer cells resistance to MST1/2-dependent apoptosis
}

\author{
S. Pauliina Turunen ${ }^{1}$ - Pernilla von Nandelstadh ${ }^{2} \cdot$ Tiina Öhman $^{3}$ - Erika Gucciardo ${ }^{2}$ - Brinton Seashore-Ludlow ${ }^{4}$. \\ Beatriz Martins ${ }^{2} \cdot$ Ville Rantanen ${ }^{2} \cdot$ Huini $\mathrm{Li}^{1} \cdot$ Katrin Höpfner $^{2} \cdot$ Päivi Östling $^{4} \cdot$ Markku Varjosalo $^{3} \cdot$ Kaisa Lehti $^{1,2}$
}

Received: 2 October 2018 / Revised: 18 February 2019 / Accepted: 7 March 2019 / Published online: 22 March 2019

(c) The Author(s) 2019. This article is published with open access

\begin{abstract}
Cancer cells balance with the equilibrium of cell death and growth to expand and metastasize. The activity of mammalian sterile20-like kinases (MST1/2) has been linked to apoptosis and tumor suppression via YAP/Hippo pathway-independent and -dependent mechanisms. Using a kinase substrate screen, we identified here MST1 and MST2 among the top substrates for fibroblast growth factor receptor 4 (FGFR4). In COS-1 cells, MST1 was phosphorylated at Y433 residue in an FGFR4 kinase activity-dependent manner, as assessed by mass spectrometry. Blockade of this phosphorylation by Y433F mutation induced MST1 activation, as indicated by increased threonine phosphorylation of MST1/2, and the downstream substrate MOB1, in FGFR4-overexpressing T47D and MDA-MB-231 breast cancer cells. Importantly, the specific knockdown or short-term inhibition of FGFR4 in endogenous models of human HER2 ${ }^{+}$breast cancer cells likewise led to increased MST1/ 2 activation, in conjunction with enhanced MST1 nuclear localization and generation of N-terminal cleaved and autophosphorylated MST1. Unexpectedly, MST2 was also essential for this MST1/N activation and coincident apoptosis induction, although these two kinases, as well as YAP, were differentially regulated in the breast cancer models analyzed. Moreover, pharmacological FGFR4 inhibition specifically sensitized the HER2 ${ }^{+}$MDA-MB-453 breast cancer cells, not only to HER2/EGFR and AKT/mTOR inhibitors, but also to clinically relevant apoptosis modulators. In TCGA cohort, FGFR4 overexpression correlated with abysmal HER2 $2^{+}$breast carcinoma patient outcome. Therefore, our results uncover a clinically relevant, targetable mechanism of FGFR4 oncogenic activity via suppression of the stress-associated MST1/2induced apoptosis machinery in tumor cells with prominent HER/ERBB and FGFR4 signaling-driven proliferation.
\end{abstract}

These authors contributed equally: S. Pauliina Turunen, Pernilla von Nandelstadh

\section{Edited by R. A. Knight}

Supplementary information The online version of this article (https:// doi.org/10.1038/s41418-019-0321-x) contains supplementary material, which is available to authorized users.

\section{Kaisa Lehti}

kaisa.lehti@ki.se

1 Department of Microbiology, Tumor and Cell Biology (MTC), Karolinska Institutet, Stockholm SE-171 77, Sweden

2 Research Programs Unit, Genome-Scale Biology, Medicum, University of Helsinki and Helsinki University Hospital, Helsinki FI-00014, Finland

3 Institute of Biotechnology, Helsinki Institute of Life Science, University of Helsinki, Helsinki FI-00014, Finland

4 Department of Oncology and Pathology, Science for Life Laboratory, Karolinska Institutet, Stockholm SE-171 77, Sweden

\section{Introduction}

Cancer cells rely on oncogenic signaling by receptor tyrosine kinases (RTKs) to drive tumor initiation and progression [1]. Upon tumor evolution, RTKs contribute to the development of resistance toward initially effective anticancer treatments. Owing to inhibitable enzyme activity and cell surface localization, RTKs thus serve as attractive therapy targets. Among RTKs, fibroblast growth factor receptors (FGFRs) trigger intracellular signaling cascades that control key cellular processes including survival, proliferation, differentiation, and migration/invasion, as well as angiogenesis-each dysregulated in cancer [2]. Four homologous FGFRs are expressed in humans [2]. Out of these, FGFR4 is dispensable for mouse development [3]. This coupled with specific FGFR4 induction in certain cancers, along with structural differences and drug selectivity relative to other FGFRs, supports the efficacy of FGFR4 as a therapeutic intervention [4-9]. 
Despite advances in diagnosis and treatment, subsets of breast cancer remain challenging to cure accounting for an estimated $15 \%$ of cancer deaths in women [10]. As in other cancers, the poor prognosis is partially attributed to therapy resistance and anti-apoptosis responses of the cancer cells [11]. FGFR4 overexpression and gene alterations, including G388R single-nucleotide polymorphism, have been associated with cancer invasion, drug resistance, and poor prognosis [12-14]. In breast cancers, FGFR4 is overexpressed in especially ERBB2/HER2-enriched tumors [4], where it has been linked to tumor growth and apoptosis resistance [4, 5, 15]. Acquired alterations have been reported in FGFR4 and ERBB2/HER2 upon metastatic cancer progression [16]. Despite these results, the molecular mechanisms explaining how FGFR4 confers aggressive cancer cell behavior remain incompletely understood.

Here, we screened for FGFR4 substrates using in vitro kinase substrate microarrays. Unexpectedly, Hippo tumor suppressor pathway components, including the serine/ threonine kinases MST1/2 (mammalian sterile20-like kinases), were among the top tyrosine-phosphorylated substrates. Cytoplasmic MST1/2 comprises the core mammalian Hippo pathway kinase complex, which activation ultimately leads to serine phosphorylation-dependent cytoplasmic retention and inactivation of the oncogenic transcriptional regulators, YES-associated protein (YAP1), and transcriptional co-activator with PDZ-binding motif (TAZ) [17, 18]. Upon cell stress and apoptosis, caspase-3 cleavage removes the inhibitory C-terminal domains of autophosphorylated MST1/2 to induce transport of activated N-terminal MST1/2 into the nucleus [19]. Although overexpression results have shown that nuclear MST1/2 can promote apoptosis [20-23], and reduced MST1/2 activity associates with poor cancer prognosis [24-27], the functional contributions of endogenous MST1/2 in physiological or pathological apoptosis remain elusive [19]. Our screening results led us to investigate in more detail the functional interaction of FGFR4 and MST1/2. Altogether, our results establish a unique oncogenic signaling mechanism - the dominant FGFR4-mediated attenuation of MST1/2-mediated, stress-associated apoptosis in HER2 ${ }^{+}$ breast cancer cells.

\section{Materials and methods}

\section{Kinase substrate identification array}

For in vitro FGFR4 substrate identification, the protein microarray containing 9483 human recombinant proteins (Protoarray human protein microarrays version 5.0; Invitrogen, Carlsbad, CA, USA) was blocked and incubated with the recombinant kinase domain of FGFR4 (50 nM;
Invitrogen) in the presence of $[\gamma-33 \mathrm{P}]$ ATP. The array was washed to remove unbound $\gamma-33 \mathrm{P}$, and exposed to X-ray film. The acquired array image was analyzed using ProtoArray Prospector software (Invitrogen). The raw data were subjected to background subtraction, signal scatter compensation, and outlier detection. The Z-factor cutoff value was set at $\geq 0.4$. Phosphorylated proteins with a Z-score $>$ 0.25 were considered as potential substrates.

\section{Cell culture}

Human ZR-75.1, MCF7, BT474, T47D, MDA-MB-453, Hs578T, BT549, MDA-MB-231 (American Type Culture Collection; ATCC, Manassas, VA, USA) and SUM159 [28] breast carcinoma cells, and COS-1 cells (ATCC) were cultured according to manufacturer's instructions. The MycoAlertPlus kit (LT07-705, Lonza, Basel, Switzerland) was used for confirming the cell cultures negative for mycoplasma.

\section{Antibodies, inhibitors, and growth factors}

The antibodies used were as follows: mouse monoclonal antibodies against ERBB2/HER2 (MA5-13105, Thermo Fisher Scientific (Waltham, MA, USA) for IF, and NCL-LCB11; Leica Biosystems, Wetzlar, Germany), FGFR4 (sc136988, Santa Cruz Biotechnology, Dallas, TX, USA), FLAG (9A3; 8146, Cell Signaling Technology, Beverly, MA, USA), glyceraldehyde-3-phosphate dehydrogenase (G8795; Merck, Darmstadt, Germany), MST1 (sc-100449, Santa Cruz Biotechnology, for IF), phospho-tyrosine (05321, Merck), YAP/TAZ (63.7; sc-101199, Santa Cruz Biotechnology), rabbit monoclonal antibodies against active YAP (EPR19812; ab205270, Abcam, Cambridge, UK), and MOB1 (13730 S), MST1 (D8B9Q; 14946), phosphoMST1/2 (T183/180) (E7U1D; 49332), phospho-MOB1 (pT35) (D2F10; 8699), and phospho-tyrosine (8954, MultiMab) all from Cell Signaling Technology. Rabbit polyclonal antibodies against FGFR4 (sc-124; Santa Cruz), phospho-FGFR4 (pY642; CSB-PA008250, Cusabio Technology, Houston, TX, USA) and phospho-FRS2- $\alpha$ (pY196; 3864), MST1 (3682), MST2 (3952), phospho-p44/42 MAPK (phospho-Erk1/2) (pT202/pY204; 9101), phosphoAKT (pS473; 9271), phospho-MST1/2 (pT183/pT180; 3681), phospho-YAP (pS127; 4911) all from Cell Signaling Technology, V5-tag (ab9116; Abcam), and horseradish peroxidase-conjugated secondary antibodies (P044701 and P044801, Dako, Santa Clara, CA, USA) for enhanced chemiluminescence detection of immunoblots. Speciesmatched Alexa Fluor conjugated secondary antibodies and phalloidin (Life Technologies, Carlsbad, CA, USA) were used for immunofluorescence. FGFR4 inhibitor BLU9931 (S7819) [29] was purchased from Selleckchem (Munich, 
Germany), and MST1/2 inhibitor XMU-MP-1 (6482) [30] from Tocris Bioscience (Bristol, UK). Okadaic acid (10011490) was purchased from Cayman Chemical (Ann Arbor, MI, USA). Recombinant human fibroblast growth factor 1 (GF002) and fibroblast growth factor 2 (GF003) were purchased from Merck.

\section{CDNA constructs, small interfering RNAs, and short hairpin RNAs}

Flag epitope (N-terminal)-tagged MST1 and MST2 in the p3FLAG-CMV-10wt (Merck) expression vectors have been described previously [31]. MST1-Y433F mutant was generated by site-directed mutagenesis. FGFR4 and its kinase activity-deficient mutant $(\mathrm{K} 503 \mathrm{M})$ were cloned into pcDNA3.1/V5-His vector, and the expression vector for FGFR4 G388 variant was generated from R388 variant by site-directed mutagenesis [32, 33]. Pools of four siRNAs against human STK3 (MST2; L-004874), STK4 (MST1; L004157), FGFR4 (L-003134), and nontargeting control siRNA (D-001206-14) (all from Dharmacon/Horizon Discovery, Cambridge, UK) were used; and for FGFR4 also SI02665306 (siFGFR4_6), SI00031360 (siFGFR4_2) and SI00031374 (siFGFR4_4) from Qiagen (Hilden, Germany).

Short hairpin RNA (shRNA) targeted against FGFR4 (TRCN0000000628 and TRCN0000199510; or nontargeting scrambled shRNA were used (Dharmacon/Horizon Discovery). The packaging plasmid (pCMVdr8.74), envelope plasmid (pMD2-VSVG), and FGFR4 or scrambled shRNA in pLKO.1 vector were co-transfected into 293FT producer cells using Lipofectamine 2000 reagent (Invitrogen). Complete breast carcinoma cell growth medium was changed on 293FT cells $24 \mathrm{~h}$ after transfection. The viral supernatants were collected after $48 \mathrm{~h}$, passed through a $0.45-\mu \mathrm{m}$ filter, and incubated with human breast carcinoma cells. After $16 \mathrm{~h}$ of infection, the supernatants were replaced with complete media followed by puromycin $(2-5 \mu \mathrm{g} / \mathrm{ml})$ selection of the transduced cells [34].

\section{Cell transfections, sphere preparation, and treatments}

The cells were transfected with expression vectors using FuGENE HD (Promega, Madison, WI) and siRNAs using Lipofectamine 2000 (Invitrogen), Interferin (Polyplustransfection SA, Illkirch-Graffenstaden, France) or Dharmafect 1 or Dharmafect 2 (Dharmacon/Horizon Discovery). For 2D immunofluorescence, cells were seeded on monomeric collagen type I-coated $(50 \mu \mathrm{g} / \mathrm{ml}, \mathrm{C} 7661$, Merck) coverslips, fixed in $4 \%$ paraformaldehyde ( $\mathrm{pH} 7.5$ ), and stained as previously described [35], and mounted in
Vectashield with (DAPI; Vector Laboratories, Burlingame, CA, USA).

Spheres of 24,000 cells were allowed to form under nonadherent conditions in agarose-coated 96-well plates in cell culture media supplemented with $0.2 \mathrm{mg} / \mathrm{ml}$ Matrigel (growth factor reduced, 354230; Corning/Merck), or on ultra-low attachment 96-well plate (Corning ${ }^{\circledR}$ Costar $^{\circledR}$ CLS7007, Merck) without Matrigel, and cultured for 2 8 days [36]. The spheres were cultured in the indicated serum concentrations, or serum-starved for $16 \mathrm{~h}$ before inhibitor treatment, followed by lysis with radioimmunoprecipitation assay (RIPA) buffer. For immunofluorescence, the spheres were fixed in $4 \%$ paraformaldehyde ( $\mathrm{pH} 7.5$ ), post-fixed in ice-cold acetonemethanol (1:1) solution, incubated in blocking buffer $(5 \%$ bovine serum albumin (BSA), $0.1 \%$ Triton X-100 in PBS) and stained for MST1 and YAP. Nuclei were visualized with Hoechst 33342 stain (Thermo Fisher Scientific). The spheres were mounted on glass slides in Vectashield (Vector Laboratories). At least two independent cultures per stable lentiviral shRNA transduction were analyzed for MST1.

\section{Immunoprecipitation and immunoblotting}

Immunoprecipitation and immunoblotting were performed as described previously [37]. Cells were lysed with RIPA buffer (50 mm Tris-HCl pH 7.4, $150 \mathrm{~mm} \mathrm{NaCl,} 1 \%$ Igepal CA-630, 0.5\% sodiumdeoxycholate) containing Complete protease inhibitor cocktail (Merck), $2 \mathrm{mM} \mathrm{Na}_{3} \mathrm{VO}_{4}$, and $2 \mathrm{~mm}$ $\mathrm{NaF}$ and cleared by centifugation. For immunoprecipitation from the soluble cell lysates, the samples were further diluted 1:3 with Triton lysis buffer (50 mM Tris- $\mathrm{HCl} \mathrm{pH} \mathrm{8.0,}$ $150 \mathrm{~mm} \mathrm{NaCl}, 1 \%$ Triton X-100, $5 \mathrm{~mm} \mathrm{CaCl}_{2}, 0.02 \% \mathrm{NaN}_{3}$ ) containing Complete protease inhibitor cocktail, $2 \mathrm{~mm}$ $\mathrm{Na}_{3} \mathrm{VO}_{4}$, and $2 \mathrm{~mm} \mathrm{NaF}$ and pre-cleared with protein $\mathrm{A}$ Sepharose. MST1 or MST2 were immunoprecipitated from cleared supernatants with EZview Red anti-FLAG M2 Affinity Gel (Merck) for $4 \mathrm{~h}$ at $4{ }^{\circ} \mathrm{C}$. After washing with Triton lysis buffer, bound proteins were eluted with reducing sodium dodecyl sulphate (SDS-PAGE) sample buffer $(0.12 \mathrm{M}$ Tris- $\mathrm{HCl} \mathrm{pH} 6.8,0.02 \%$ bromophenol blue, $4 \%$ SDS, $50 \%$ glycerol, $0.1 \mathrm{~m}$ dithiotreitol). Protein concentrations were determined with BCA Protein Assay Kit (23225, Thermo Fischer Scientific), and equal protein amounts ( $\mu \mathrm{g})$ were separated by SDS-PAGE and transferred to nitroclellulose membranes for subsequent detection with primary antibodies and matched secondary antibodies conjugated to horseradish peroxidase. All immunoblots are representative images of experiments repeated independently at least three times; except for Figs. 5b, e and 6a, b, where the experiments were repeated twice. 
Mass spectrometry analysis of phosphorylation sites

All buffers of immunoprecipitation and elution procedures were supplemented with Complete protease inhibitor cocktail and PhosSTOP phosphatase inhibitor tablets (Merck). After the general immunoprecipitation washes, the anti-FLAG affinity gel was washed with pre-urea wash buffer $(50 \mathrm{~mm}$ Tris $\mathrm{pH} 8.5,1 \mathrm{~mm}$ ethylene glycol-bis $(\beta-$ aminoethyl ether (EGTA), $75 \mathrm{~mm} \mathrm{KCl}$ ) before elution with urea buffer ( $6 \mathrm{~m}$ urea, $20 \mathrm{~mm}$ Tris $\mathrm{pH}$ 7.5, $100 \mathrm{~mm}$ sodium chloride). Elution cycles were repeated three times for 30 min each at room temperature with agitation. Proteins in eluates were reduced with (tris(2-carboxyethyl)phosphine), alkylated with iodoacetamide, and trypsin digested with Sequencing Grade Modified Trypsin (Promega). Phosphopeptide enrichment was performed using immobilized metal ion affinity chromatography with titanium (IV) ion $\left(\mathrm{Ti}^{4}\right.$ ${ }^{+}$-IMAC). IMAC material was prepared and used essentially as described [38]. The liquid chromatography with tandem mass spectrometry (LC-MS/MS) analysis was performed using a Q Exactive ESI-quadrupole-orbitrap mass spectrometer coupled to an EASY-nLC 1000 nanoflow LC (Thermo Fisher Scientific), using Xcalibur version 3.1.66.10 (Thermo Fisher Scientific). The phoshopeptide sample was loaded from an autosampler into a C18-packed precolumn (Acclaim PepMap ${ }^{\mathrm{TM}} 100 ; 100 \mu \mathrm{m} \times 2 \mathrm{~cm}, 3 \mu \mathrm{m}$, $100 \AA$ A, Thermo Fisher Scientific) in buffer A (1\% acetonitrile, $0.1 \%$, formic acid). Peptides were transferred onward to a C18-packed analytical column (Acclaim PepMap $^{\mathrm{TM}} 10075 \mu \mathrm{m} \times 15 \mathrm{~cm}, 2 \mu \mathrm{m}, 100 \AA$, Thermo Fisher Scientific) and separated with 60-minute linear gradient from 5 to $35 \%$ of buffer B (98\% acetonitrile, $0.1 \%$ formic acid) at the flow rate of $300 \mathrm{nl} /$ minute. The MS analysis was performed in data-dependent acquisition in positive ion mode. MS spectra were acquired from m/z 300 to $\mathrm{m} / \mathrm{z} 2000$ with a resolution of 70,000 with Full AGC target value of $3,000,000$ ions, and a maximal injection time of $120 \mathrm{~ms}$, in profile mode. The ten most abundant ions with charge states from $2+$ to 7 + were selected for subsequent fragmentation (higher energy collisional dissociation, HCD) and MS/MS spectra were acquired with a resolution of 17,500 with AGC target value of 5000, a maximal injection time of $120 \mathrm{~ms}$, and the lowest mass fixed at $\mathrm{m} / \mathrm{z} 120$, in centroid mode. Dynamic exclusion duration was $30 \mathrm{~s}$. Raw data files were analyzed with the Proteome Discoverer software version 1.3 (Thermo Fisher Scientific) connected a Sequest search engine version 28.0 (Thermo Fisher Scientific) against the human component of the Uniprot Database (version 07_2017). Carbamidomethylation (+57.021 Da) of cysteine residues was used as static modification. Phosphorylation of Ser/Thr/Tyr $(+79.966 \mathrm{Da})$ and oxidation $(+15.994 \mathrm{Da})$ of methionine was used as dynamic modification. Precursor mass tolerance and fragment mass tolerance were set to $<15$ ppm and $0.05 \mathrm{Da}$, respectively. A maximum of two missed cleavages was allowed. The software phosphoRS [39] was used to calculate the indvidual site probabilities for phosphorylated peptides.

\section{Flow cytometry analysis of apoptosis}

The proportions of apoptotic cells were determined by flow cytometry for annexin $\mathrm{V}$ and propidium iodide (PI) binding [40]. Stable shScr or shFGFR4 transduced MDA-MB-453 cells were transfected with siRNAs. After $72 \mathrm{~h}$ the cells, including floating cells in the medium, were collected by trypsinization, washed and labeled with annexin V-Alexa Fluor488 (A13201; Life Technologies) diluted into binding buffer (10 mM HEPES-140 mm sodium chloride- $2.5 \mathrm{~mm}$ calcium chloride) for $15 \mathrm{~min}$ at room temperature. Cells were suspended into binding buffer, stained with $1 \mu \mathrm{g} / \mathrm{ml}$ PI (P3566, Life Technologies), and analyzed with BD Accuri C6 flow cytometer (BD Biosciences, San Jose, CA, USA). Gating and data analysis were performed using FlowJo v10.1 software (Tree Star Inc., Ashland, OR, USA). Geometric mean fluorescence of annexin $\mathrm{V}$ binding (FL1-A) alone or combined with PI binding (FL3-A) was quantified. Experiments were repeated three times.

\section{In vitro kinase assays}

MST1 Kinase Enzyme System, including Axltide peptide (KKSRGDYMTMQIG) as MST1 substrate, with ADP-Glo reagent (V4153, Promega) was used according to manufacturer's instructions to measure ADP production in kinase reactions. The reaction buffer $(40 \mathrm{~mm}$ Tris, $\mathrm{pH} 7.5-20 \mathrm{~mm}$ magnesium chloride $-0.1 \mathrm{mg} / \mathrm{ml} \mathrm{BSA}-50 \mu \mathrm{M}$ dithiotreitol) was supplemented with $2.5 \mu \mathrm{M}$ manganese chloride to enhance tyrosine kinase activity. Recombinant MST1 kinase ( $3 \mathrm{ng}$ ) was incubated with $0-10 \mathrm{ng}$ of recombinant kinase domain of FGFR4 (P3054, Thermo Fisher Scientific), together with $1 \mu \mathrm{M}$ XMU-MP-1 (MST1/2 inhibitor) as an assay control. The kinase reactions with final $50 \mu \mathrm{M}$ ATP concentration were incubated at room temperature for $1 \mathrm{~h}$ on ProxiPlate (PerkinElmer, Waltham, MA, USA), ADPGlo reagent was added for $40 \mathrm{~min}$, followed by addition of kinase detection reagent for $30 \mathrm{~min}$ ' incubation before reading the luminescence with EnSight plate reader (PerkinElmer). Experiment was repeated three times. For in vitro kinase assay detection by immunoblotting, $40 \mathrm{ng}$ of MST1 kinase and 0-100 ng recombinant kinase domain of FGFR4 were incubated together for $30 \mathrm{~min}$ at $30^{\circ} \mathrm{C}$, with or without $1 \mu \mathrm{M}$ XMU-MP-1 or $100 \mathrm{~nm}$ BLU9931 (FGFR4 inhibitor) using the abovementioned buffers and MST1 substrate. Proteins in the kinase reaction were denatured and reduced using SDS-PAGE sample buffer $(0.12 \mathrm{M}$ Tris- $\mathrm{HCl} \mathrm{pH} 6.8,0.02 \%$ bromophenol blue, $4 \%$ 
SDS, $50 \%$ glycerol) supplemented with $10 \%(\mathrm{v} / \mathrm{v}) \beta$-mercaptoethanol, and subjected to immunoblotting for detecting phosphorylation of MST1 and FGFR4. Experiment was repeated two times.

\section{Drug sensitivity and resistance testing and data processing}

Compounds (from FIMM Oncology set; https://www.fimm. fi/en/services/technology-centre/htb/equipment-and-libra ries/chemical-libraries) and viability controls (DMSO, $100 \mu \mathrm{m}$ benzethonium chloride) were predispensed on tissue culture treated 384-well plates (Corning, NY, USA). Each compound was plated as singlicate in five concentrations spanning a 10,000-fold concentration range (10-fold dilution). Assay ready plates were stored in pressurized StoragePods (Roylan Developments, Surrey, UK) under inert atmosphere until used. Using a MultiDrop Combi (Thermo Scientific) $5 \mu \mathrm{L}$ media with or without $500 \mathrm{~nm}$ BLU9931 (5x concentration) was first dispensed into assay ready plates and centrifuged briefly. Twenty microliters of a single-cell suspension (2000 cells) was then seeded using MultiDrop Combi peristaltic pump to the plates, which were centrifuged briefly and transferred to an incubator $\left(37^{\circ} \mathrm{C}\right.$ and $5 \% \mathrm{CO}_{2}$ ). As a surrogate for cell viability, cellular ATP levels were assessed $72 \mathrm{~h}$ after plating using CellTiterGlo 2.0 (Promega) with detection on an EnSight plate reader (PerkinElmer). Using in house software, data from the plate reader were normalized per plate to percent viability using values from control wells. Concentration-response curves were fitted to percent viability values using a four-parameter logistic model, and processed further to a sensitivity metric (drug sensitivity score; DSS) using a weighted area-underthe-curve calculation [41]. DSS for each drug was compared between conditions tested.

\section{Real-time quantitative PCR (qPCR)}

RNA was extracted with an RNeasy Plus Mini kit (Qiagen), coupled with RNeasy MinElute columns (Qiagen) for small amount of starting material from non-adherent sphere cultures, followed by reverse transcription with Maxima First Strand cDNA Synthesis Kit (Thermo Scientific). mRNA expression was quantified using TaqMan Fast Advanced Master Mix (Applied Biosystems, Foster City, CA, USA), and validated primers (connective tissue growth factor (CTGF), Hs01026927_g1; cysteine rich angiogenic inducer 61 (CYR61), Hs_00998500_g1; ankyrin repeat domain 1 (ANKRD1), Hs_00173317_m1; TATA-binding protein (TBP) Hs99999910_m1; Applied Biosystems). The expression was normalized with TBP mRNA expression. Two independent RNA extractions were performed, each analyzed in triplicates by qPCR.

\section{Fibrin-embedded 3D cell cultures for immunohistochemisrty}

Preparation of cross-linked fibrin gels was perfomed essentially as described [42], and 17,250 cells were suspended into $75 \mu \mathrm{l}$ of fibrin gel and casted to 96-well plate. Matrix-embedded single cells were cultured with complete growth medium atop for 7-8 days before first treatment with $100 \mathrm{~nm}$ BLU9931 and/or $30 \mathrm{ng} / \mathrm{ml}$ FGF1. Inhibitor treatment was added again $72 \mathrm{~h}$ later, and cells were fixed after 13-14 days of culture with 4\% paraformaldehyde in PBS for $4 \mathrm{~h}$ at room temperature. Fibrin gels were dehydrated, paraffin-embedded for sectioning, and subjected immunohistochemistry essentially as described [43]. Sections were subjected to heat-induced antigen retrieval in Tris-ethylenediaminetetraacetic acid, $\mathrm{pH} 9.0$ (for Ki67 antibody, ACK02, Leica Biosystems), or in sodium citrate, pH 6.0 (for Bax antibody, HPA027878, Sigma-Aldrich). Sections were subsequently incubated in $3 \%$ (v/v) hydrogen peroxide for $10 \mathrm{~min}$. For antigen detection, tyramide signal amplification (TSA ${ }^{\mathrm{TM}}$, PerkinElmer) technology, followed by incubation with 3-amino-9-ethyl-carbazole solution, were used according to manufacturer's instructions. Total 30 colonies from two replicate samples were quantified for positively stained vs. total number of cells.

\section{Imaging and image quantification}

Fluorescence images were obtained using an AxioImager. Z2 upright epifluorescence microscope with PlanApochromat $\times 20 / 0.8$ NA dry or $\times 40 / 1.4$ NA oil objective. In addition, an LSM 780 confocal microscope with Plan-Neofluar $\times 40 / 1.3$ NA oil objective was used (all from Carl Zeiss, Oberkochen, Germany). Brightness and contrast were linearly adjusted using ZEN 2012 (blue edition; Carl Zeiss). Single optical sections or a combination of two serial optical sections were used for image display.

The MST1 and YAP-stained sphere images were analyzed using Anima [44]. Cell nuclei were detected from 4',6-diamidino-2-phenylindole (DAPI) channel by first normalizing images with adaptive histogram equalization, and then using the Shape filtering segmentation method found in Anima. The MST1 or YAP signal intensity values were measured from the nucleus area and from a ring around the nucleus representing the cytoplasm. The ring width was determined as $50 \%$ of the radius of the nucleus. The nucleus/cytoplasm intensity ratio was calculated by dividing nuclear intensity with cytoplasmic intensity for each nucleus, and then calculating a median value for each image.

Image quantifications of western blotting were performed by processing all obtained micrographs with ImageJ software. 
Fig. 1 FGFR4 substrate screen identifies tyrosine-

phosphorylated Hippo pathway proteins including MST1/2.

a Scheme of the substrate screen with recombinant FGFR4 kinase domain. b Top 10 FGFR4 substrates ranked by the Z-score include Hippo pathway -associated proteins (yellow). See Table S1 for the full substrate list. c, d MST1/2 are tyrosine phosphorylated by FGFR4 in COS-1 cells. Flagtagged MST1/2 were immunoprecipitated after transfection of MST1 and MST2 alone or in combination with FGFR4 G388 (G), or R388 (R) kinase (wt), or kinase-dead (KD) variants, and detected by immunoblotting. e MST1 immunoprecipitates from COS-1 cells co-transfected with FGFR4 (R)-wt or FGFR4 (R)-KD (See Fig. S1A) were trypsin digested and subjected to phoshopeptide enrichment prior to LC-MS/MS analysis $(N=3)$ that identified phosphorylated Y433 (red) on MST1 only with FGFR4 (R)-wt, and phosphorylated S410 (green) only with FGFR4 (R)KD
A

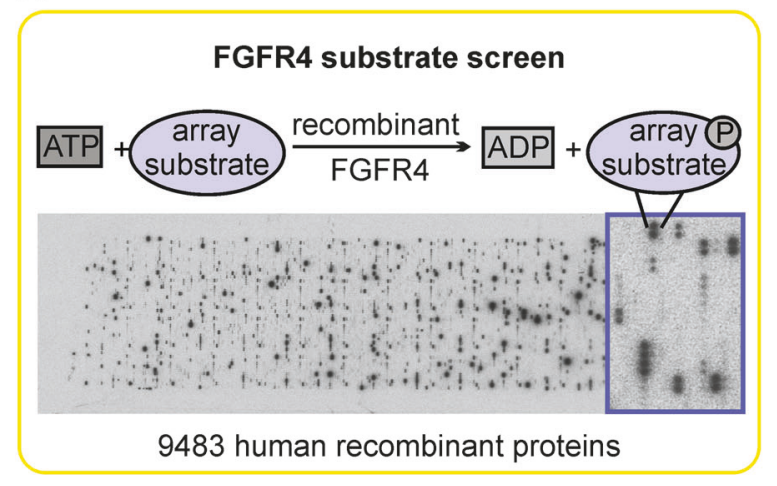

B

C

MST1-Flag $+++\ldots$.

MST2-Flag - - + + + - -

FGFR4-V5 - GR - GR GR
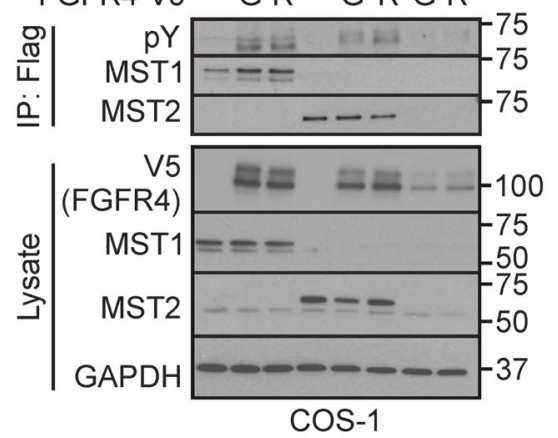

cos-1

410

D

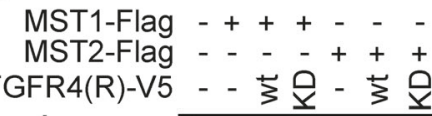
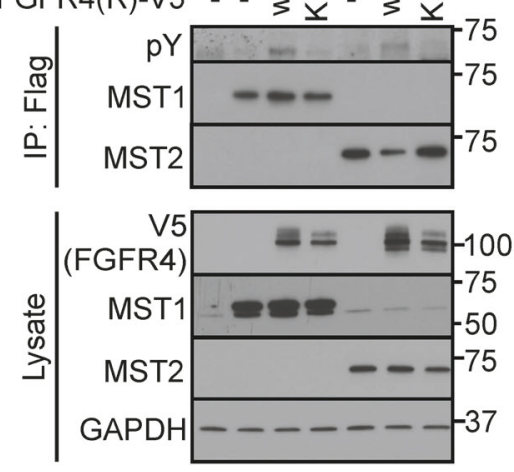

COS-1

E

433

\section{Statistics}

All numerical values represent mean \pm SD or SEM as indicated in figure legends. Statistical significance was determined using two-tailed Student's $t$ tests for analysis of apoptosis, quantifications of immunoblots and immunohistochemistry. The Kolmogorov-Smirnov test was used for computerized image analysis data on MST1 and YAPstained sphere images.

\section{Results}

\section{FGFR4 tyrosine phosphorylates Hippo pathway proteins in vitro and in cells}

To systematically screen for substrates serving as downstream effectors of FGFR4, we used recombinant FGFR4 kinase domain to assess in vitro phosphorylation of 9483 human recombinant proteins (Fig. 1a, Table S1, Z-score ranking). Unexpectedly, the top five substrates included four Hippo tumor suppressor pathway proteins; MST2 (STK3), protein kinase $\mathrm{C}$ iota (PRKCI), casein kinase I delta (CSNK1D) [45], and MST1 (STK4) (Fig. 1b; STK3 and PRKCI identified as two splice variants), suggesting that FGFR4 can directly phosphorylate the Hippo serine/threonine kinase pathway proteins (Fig. 1b). To validate this activity, MST1/2 were immunoprecipitated from COS-1 cells after transfection of MST1 and MST2 alone or in combination with FGFR4-G (G388) or the cancerassociated FGFR4-R (R388) variant. Notably, both variants induced MST1 and MST2 tyrosine phosphorylation, revealing these Hippo kinases as novel FGFR4 substrates (Fig. 1c). Importantly, the FGFR4-R-mediated MST1/2 phoshorylation was not detected with kinase activitydeficient FGFR4-R-KD (Fig. 1d).

To identify the FGFR4 phosphorylated tyrosine residue (s), immunoprecipitated MST1 was subjected to mass spectrometry (Fig. S1A). Notably, MST1 from FGFR4 expressing cells was phosphorylated at Y433, whereas MST1 from cells with inactive FGFR4 KD lacked detectable tyrosine phosphorylation (Fig. 1e, Table 1). In 
Table 1 List of MST1 phoshopeptides identified by mass spectrometry

\begin{tabular}{|c|c|c|c|c|c|}
\hline Co-transfected kinase & Protein & Phospho-residue & Peptide sequence & Peptide FDR & $\begin{array}{l}\text { phosphoRS site } \\
\text { probabilities }(\%)\end{array}$ \\
\hline \multirow[t]{4}{*}{ FGFR4/R-wt } & MST1_Q13043 & $\mathrm{Y}(433)$ & IPQDGD白FLK & $<0.01$ & 100 \\
\hline & MST1_Q13043 & $S(320)$ & 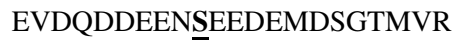 & $<0.01$ & 100 \\
\hline & MST1_Q13043 & $S(410)$ & EKENQINSFFGK & N/A & 0 \\
\hline & MST1_Q13043 & $\mathrm{T}(177)$ & LADFGVAGQLTDIMAK & $<0.01$ & 99.9 \\
\hline \multirow[t]{4}{*}{ FGFR4/R-KD } & MST1_Q13043 & $\mathrm{Y}(433)$ & IPQDGD YEFLK & N/A & 0 \\
\hline & MST1_Q13043 & $S(320)$ & EVDQDDEENSEEEDEMDSGTMVR & $<0.01$ & 100 \\
\hline & MST1_Q13043 & $S(410)$ & EKENQINSFFGK & $<0.01$ & 100 \\
\hline & MST1_Q13043 & $\mathrm{T}(177)$ & LADFGVAGQLTDTMAK & $<0.01$ & 100 \\
\hline
\end{tabular}

Fig. 2 FGFR4 is overexpressed in HER $2^{+}$, MST1 $1 / 2^{\text {low }}$ breast cancer cells. a, b FGFR4 and HER2 expression in luminal MDA-MB-453, ZR-75.1, and BT474, MCF7, and T47D, and five triple-negative breast cancer cell lines by a immunoblotting and $\mathbf{b}$ immunofluorescence. Scale bar $20 \mu \mathrm{m}$. c MST1, MST2, and YAP/TAZ expression in these cell lines, detected by immunoblotting $(N=3)$
A

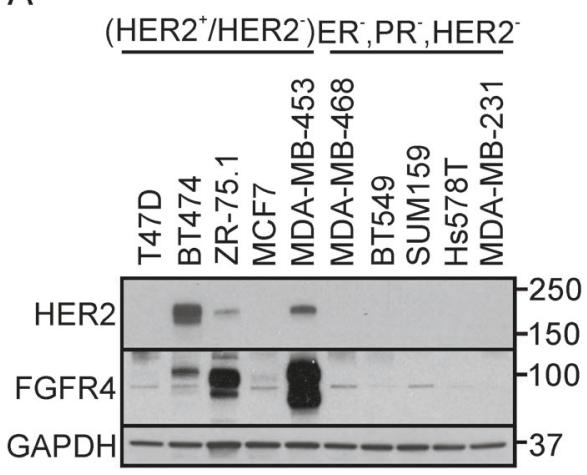

C

\section{(HER2 $\left.{ }^{+} / \mathrm{HER}^{-}\right)$ER', $\mathrm{PR}^{-}, \mathrm{HER}^{-}$}

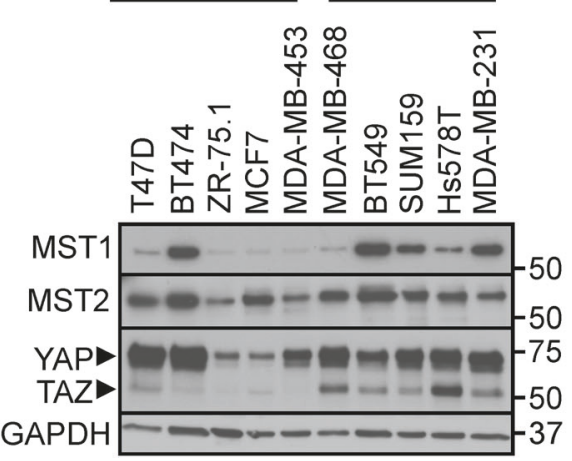

$\mathrm{B}$
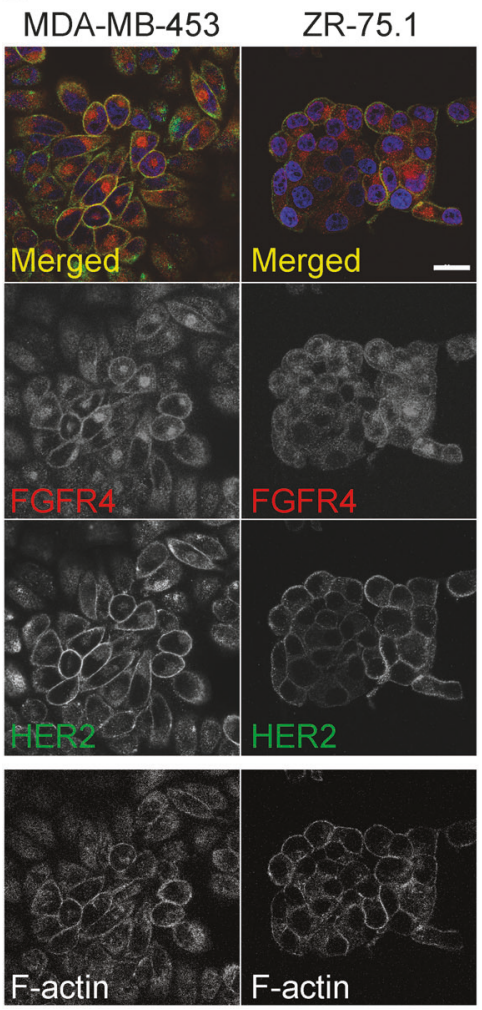

addition, an uncharacterized MST1 phosphorylation at S410 near the pY433-site was detected only with kinase-deficient FGFR4 (Fig. 1e, Table 1), whereas MST1 S320 and T177 were phosphorylated in both samples independently of FGFR4 activity (Table 1).

\section{FGFR4 is overexpressed in HER2 ${ }^{+}, \mathrm{MST}_{1} / 2^{\text {low }}$ breast cancer cells, and correlates with adverse outcome in HER2-enriched breast cancer patients}

To understand the significance of the identified novel FGFR4 activity, and to establish relevant cell models, we first analyzed FGFR4 expression using TCGA by
cBioPortal for Cancer Genomics [46, 47]. In breast cancer, FGFR4 was overexpressed in 33\% of HER2-enriched tumors (PAM50 classified, $n=58$ ) [48], and significantly associated with poorer overall survival compared with the non-overexpressing group (Fig. S1B; $P=0.044$; FGFR4 overexpression in $4 \%$ of all breast cancers; $n=825$ [4]). Consistently, MDA-MB-453 (ER $\left.{ }^{-}, \mathrm{PR}^{-}, \mathrm{HER}^{+}\right)$as well as ZR-75.1 and BT474 $\left(\mathrm{ER}^{+}, \mathrm{PR}^{+/-}, \mathrm{HER} 2^{+}\right)$breast cancer cells expressed FGFR4, whereas both FGFR4 and HER2 were low in MCF7 and T47D $\left(\mathrm{ER}^{+}, \mathrm{PR}^{+-}, \mathrm{HER}^{-}\right)$, and negligible in triple-negative $\left(\mathrm{ER}^{-}, \mathrm{PR}^{-}, \mathrm{HER} 2^{-}\right)$cells (Fig. 2a, S1C) [49]. By immunofluorescence, strong intracellular and cell surface FGFR4 was associated with cell 
surface HER2 in MDA-MB-453, which harbor an activating FGFR4 mutation [50, 51], and in ZR-75.1, rendering these cells suitable endogenous models for FGFR4 function (Fig. 2b).

Among these cell lines, MST1 was lowest in ZR-75.1, MCF7, and MDA-MB-453 (Fig. 2c). Moreover, FGFR4 ${ }^{+}$ ZR-75.1 and MDA-MB-453 expressed low levels of MST2 and TAZ, whereas TAZ was prominent in triplenegative, and YAP in all except ZR-75.1 and MCF7 cells (Fig. 2c).

\section{Endogenous FGFR4 inhibits MST1/2 activation and cleavage}

To test if FGFR4 regulates the tumor-suppressive MST1/2 kinases, MDA-MB-453 cells were transfected with siRNAs specific for FGFR4, MST1, and MST2. In control cells, weak protein bands corresponding to full length and cleaved MST1/2 were detected with antibodies against active, pT183/180-autophosphorylated MST1/2 (Fig. 3a). Markedly, FGFR4 knockdown enhanced the cleaved active pMST1/2 (Fig. 3a, arrowhead). Total MST1, present in control cells as full-length protein, became detectable as an additional $36 / 37 \mathrm{kDa}$ form after FGFR4 knockdown (Fig. 3b; see N-terminal form similar to the caspase-cleaved MST1/N; [19, 23]). MST2 instead was detected as fulllength protein (Fig. 3b). Importantly, after lentiviral FGFR4 silencing (shFGFR4), ectopic FGFR4 (R388 and G388 variant) expression rescued the cleaved MST1 and pMST1/2 back to the low levels of controls (shScr) (Fig. 3c, S2A). In contrast, cancer-associated FGFR4-R slightly induced MST2 cleavage (Fig. 3c; FGFR4-G/R vary in stability and signal output; endogenous FGFR4 in MDA-MB453 contains $\mathrm{G} 388 \mathrm{R}$ and the activating Y367C alterations [12, 33, 50-52]). In MDA-MB-231, FGFR4-R, and -G also decreased the cleaved MST1 (Fig. S2B), and even more efficiently upon FGF1 and FGF2 stimulation as shown in FGFR4-R cells (Fig. S2C).

Coincident with increased MST1/2 cleavage and activation, FGFR4 knockdown resulted in significantly increased threonine (T35) phosphorylation of the MST1/ 2 substrate MOB1 in MDA-MB-453 (Fig. 3d; $P<0.05$; [53]). In ZR-75.1, FGFR4 silencing likewise enhanced pMOB1 (Fig. 3d; $P<0.05$ ) and pMST1/2 (Fig. 3e). However, this was coupled with increased full-length and cleaved MST2, whereas cleaved MST1 was less enhanced (Fig. 3e). To clarify distinct MST contributions to FGFR4 depletion-induced pMST1/2, FGFR4 was silenced alone and in combination with MST1 or MST2 in MDA-MB-453. In subconfluent low-serum cultures with prominent activation of MST1/N upon FGFR4 depletion, partial MST1 knockdown increased pMOB1 with and without FGFR4 (Fig. 3f; note that serum suppresses distinctively MST2
[54]). Instead, MST2 double knockdown with FGFR4 blocked the enhanced threonine phosphorylation of MOB1 and cleaved MST1/2, while even increasing MST1 cleavage (Fig. 3f). Thus, MST1/2 were interregulated, and MST2 was essential for the induction of active MST1/N and pMOB1 after FGFR4 depletion.

As MDA-MB-453 and ZR-75.1 both contain the cancer-associated FGFR4-R388 (MDA-MB-453, homozygous; ZR-75.1, heterozygous), we further silenced FGFR4 in BT474 homozygous for FGFR4 G388, which increased MST1/N as well as full-length MST1/2 (Fig. 3g), indicating that both FGFR4 variants can suppress MST1.

\section{FGFR4 counteracts MST1/2-mediated apoptosis}

MST1/2 activation by T183/180 autophosphorylation and cleavage is associated with apoptosis [19]. To investigate if FGFR4 alters the pro-apoptotic MST1/2 function, MDAMB-453 cells were transduced with shScr and shFGFR4, followed by transfection of FGFR4, MST1, and MST2 siRNAs. Apoptosis was measured by annexin V and PI binding with flow cytometry using two gating strategies: P1 (smaller) and P2 (larger) cells for early apoptosis by annexin V (Fig. 4a), and the whole-cell population for combined early and late apoptotic cells by double positivity (Fig. 4b, S3B). Stable FGFR4 silencing significantly increased apoptosis relative to control (Fig. 4a, b, S3A; $P \leq$ 0.02). Similarly, FGFR4 siRNAs increased apoptosis in shScr cells (Fig. 4a, b; $P<0.001$ ). MST1 or MST2 knockdown did not decrease apoptosis in shScr cells with high endogenous FGFR4, and FGFR4 siRNAs did not further increase apoptosis in shFGFR4 cells (Fig. 4a, b). Notably, the apoptosis induction by stable FGFR4 silencing was rescued close to the low level of shScr cell apoptosis by MST1 $(P=0.02)$ or MST2 $(P=0.003)$ knockdown in shFGFR4 cells (Fig. 4a, b, and S3B), suggesting that MST1 and MST2 act together to induce apoptosis, unless attenuated by FGFR4 in MDA-MB-453.

\section{FGFR4 silencing increases MST1/2 activation and MST1 nuclear translocation in cancer cell spheres}

Three-dimensional (3D) cell spheres recapitulate the cellcell contacts and dimensionality of in vivo tumors. To investigate MST1/2 regulation in 3D, MDA-MB-453 cells were cultured in non-adherent conditions for sphere formation. In complete medium, control and FGFR4 knockdown spheres had minor differences in MST1/2 and MOB1 total protein and phoshorylation (Fig. 5a; $10 \%$ fetal bovine serum; FBS). Markedly, FGFR4 depletion in low serum increased MST1/2 cleavage (total and pT183/180) and pMOB1 (Fig. 5a; 2\% FBS). Treatment with FGFR4-specific 
A

B

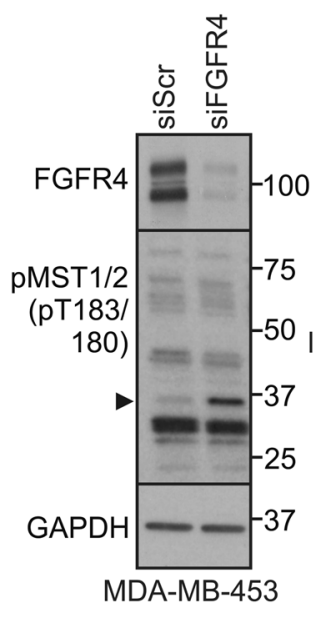

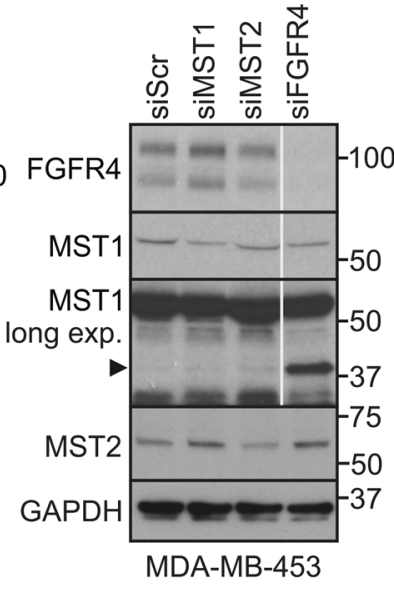

$E$

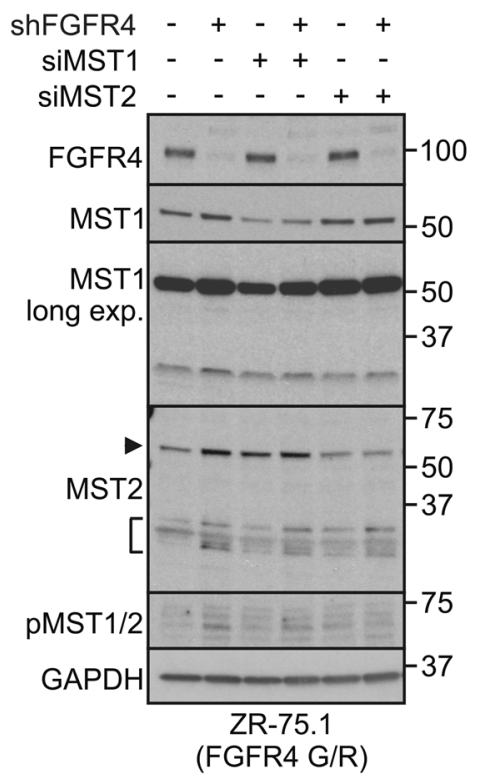

C
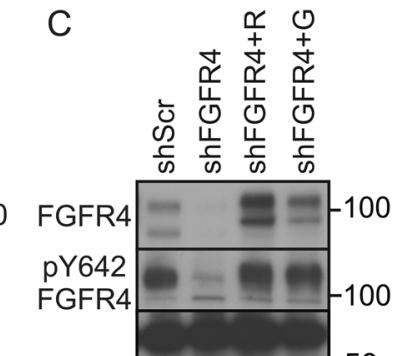

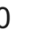

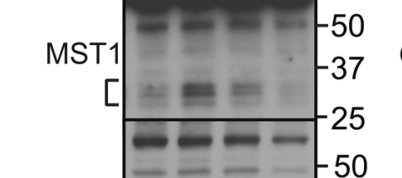

$\mathrm{D}$

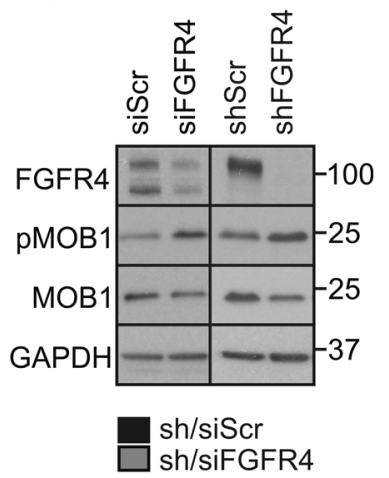

MST2

pMST1/2

(pT183/

180)

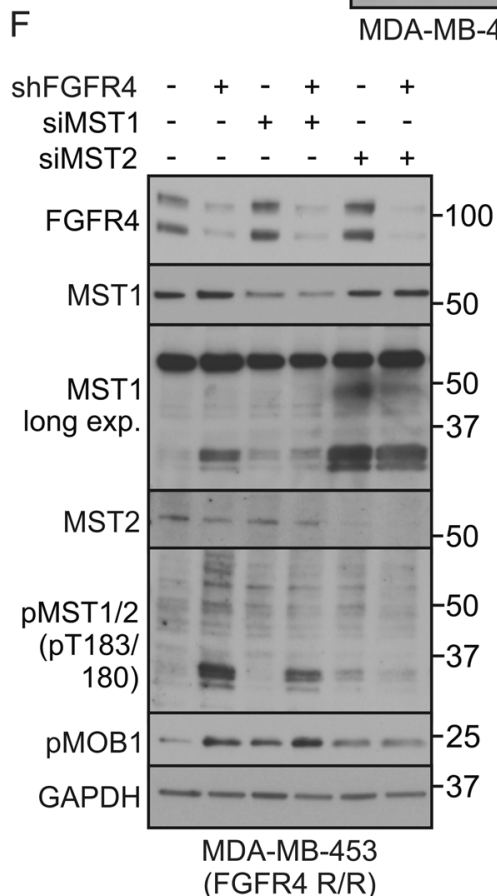

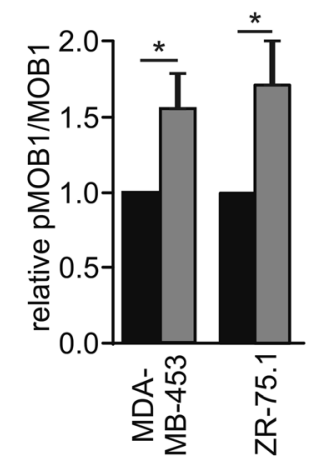
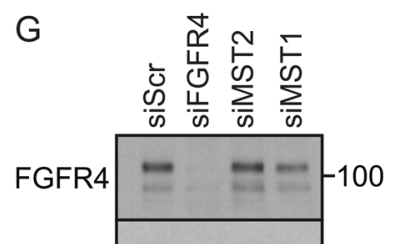

MST

MST1

long exp

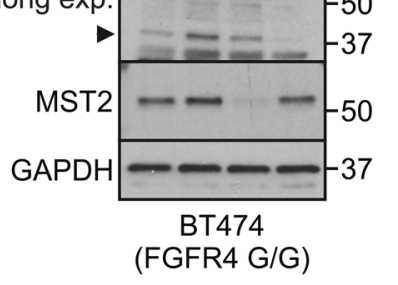

Fig. 3 FGFR4 suppresses MST1/2 activation and cleavage in HER2 ${ }^{+}$ breast cancer cells. a, b MDA-MB-453 cells transfected with indicated siRNAs were subjected to immunoblotting for a T183/180 phosphorylated MST1/2, and b MST1 and MST2. Note cleaved $~ 37 \mathrm{kDa}$ MST1/N in FGFR4 knockdown cells (arrowhead). Thin gray line indicates cropping to leave out irrelevant sample lane; see uncropped immunoblots in Fig. S8. c MDA-MB-453 cells transduced with indicated shRNAs were transfected with siScr or siFGFR4 siRNA to 3'UTR before transfection of mock or FGFR4 (R) or (G) overexpression plasmid for a rescue experiment. Lysates were subjected to immunoblotting as indicated. Brackets indicate the cleaved MST1 and
MST2 fragments. See Fig. S2A for phopsho-FRS2 $\alpha$ and short exposure of MST1. d MDA-MB-453 and ZR-75.1 cells were transduced with indicated si/shRNAs; upper, indicated immunoblots of lysates; lower, quantification of pMOB1/MOB 1 ratio, $N=3$, mean $\pm \mathrm{SEM}$; $* P<0.05$. For MST1/2 knockdown e ZR-75.1 and $\mathbf{f}$ MDA-MB-453 were transduced with shRNAs followed by transfection with siRNAs as indicated, and $\mathbf{g}$ BT474 cells were transfected with indicated siRNAs, and subjected to immunoblotting for pT183/180 MST1/2, MST1, MST2, and pMOB1 as indicated (in e arrowhead points to a full-length, bracket to the cleaved MST2) a-g. $N=3$ independent repeats for all; except $N=2$ in $\mathbf{f}$ and $\mathbf{g}$ 
A
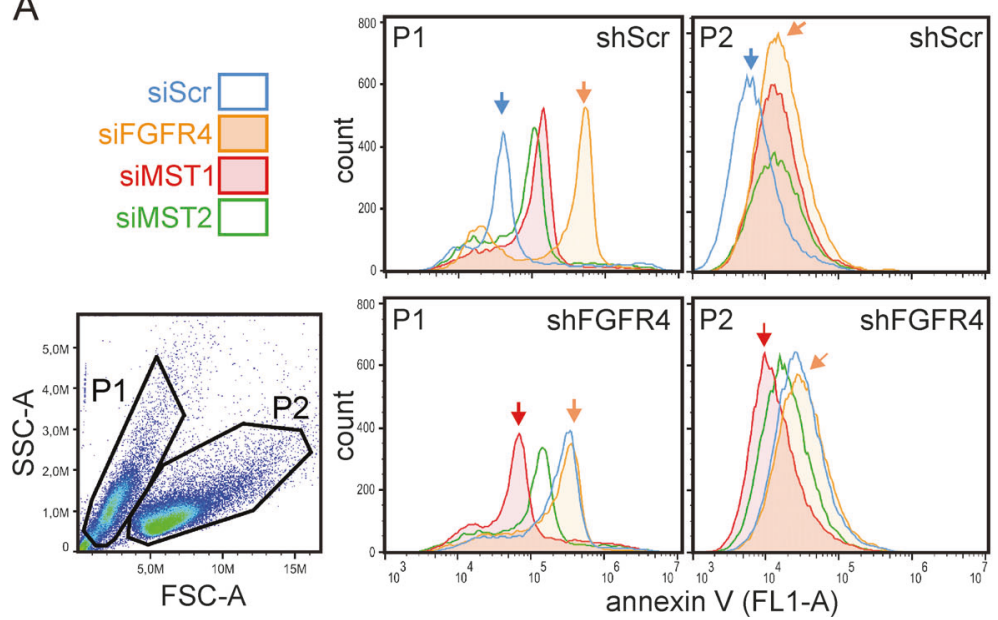

B

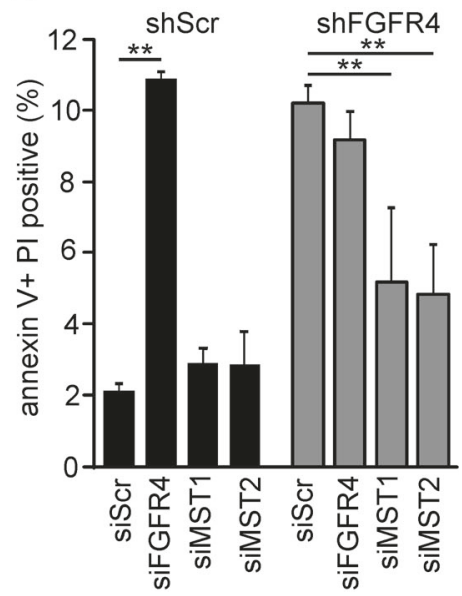

Fig. 4 FGFR4 counteracts MST1/2-mediated apoptosis. MDA-MB453 cells transduced with shScr or shFGFR4 shRNAs were transfected with siRNA pools specific for FGFR4, MST1 or MST2, and analyzed for annexin $\mathrm{V}$ and propidium iodide (PI) binding by flow cytometry using two different gating strategies for data visualization. a Gating to populations P1 (smaller) and P2 (larger), and annexin V binding
(FL1-A) histograms as a marker for early apoptotic cells. b Quantification (\% of total, 100,000 events) of apoptosis based on doublepositive (annexin $\mathrm{V}+\mathrm{PI}$ ) cells, including both early and late apoptotic stages. See Fig. S3B for representative contour plots and quadrant gating. Mean $\pm \mathrm{SD}$ of triplicates shown, $* * P<0.01$; (repeated three times; $N=3$ ). FSC-A; forward scatter, and SSC-A; side scatter inhibitor BLU9931 [29] for 15 min likewise increased pMST1/2 (Fig. 5b). During this short FGFR4 inhibition, pFRS $2 \alpha$ was diminished, whereas pAKT and pERK remained less affected (Fig. 5b), suggesting that MST1/2 activation upon FGFR4 inhibition is an early event in the apoptosis induction. By immunofluorescence, both MDAMB-453 and ZR-75.1 spheres had low nuclear and cytoplasmic MST1, whereas FGFR4 silencing specifically increased punctate, nuclear MST1, significantly increasing the nucleus/cytoplasm ratio (Fig. 5c, d, S3C), thus suggesting that FGFR4 inhibits MST1/2 activation and MST1/ $\mathrm{N}$ nuclear localization to counteract apoptosis.

To further examine the FGFR4-dependent regulation and individual MST1 and MST2 functions, all three genes were silenced in distinct combinations in 3D. As expected, FGFR4 knockdown increased the cleaved pMST1/2 and pMOB1, which response was prevented by MST1 depletion in MDA-MB-453 (Fig. 5e). After MST2 silencing alone or in combinations with FGFR4 and/or MST1, the higher molecular weight pMST1/2 forms were reduced. The cleaved pMST1/2, although reduced, remained detectable after combined MST2 and FGFR4 silencing, suggesting that it corresponds to MST1/N (Fig. 5e). However, combined knockdown of FGFR4 with MST1, MST2 or both the MSTs, prevented pMOB1 induction (Fig. 5e). In ZR-75.1 with MST2-dominated response to FGFR4 knockdown (see Fig. 3e), FGFR4 and/or MST1 knockdown instead enhanced pMOB1, whereas MST2 and FGFR4 double depletion fully prevented this induction (Fig. S4A). Therefore, MST1 and MST2 were cooperatively activated upon FGFR4 depletion, leading to context-dependent dominance of partially competing outcomes of MST2-dependent MST1/N activation or (cytoplasmic) MST2-MOB1 axis.

\section{Mutation of MST1-pY433-site restores MST1/2 activation in FGFR4 expressing cells}

To examine the effects of the identified FGFR4-dependent tyrosine phosphorylation in MST1, wild-type MST1 and the pY433-site mutant MST1-Y/F were overexpressed alone and in combination with FGFR4-R in MDA-MB-231 cells. Although weak autoactivated pMST1/2 was detected in cells overexpressing MST1 (wild-type or mutant) alone or MST1 (wild-type) and FGFR4-R, co-expression of MST1Y433F and FGFR4 resulted in prominent pMST1/2 autophosphorylation (Fig. 6a and Fig. S4B). Coincidentally, the downstream pMOB1 was increased relative to total MOB1 (Fig. 6a). Since inactivating NF2 mutation in MDA-MB231 can affect MST1/2 activation [55], we next transfected T47D cells to express FGFR4-R and MST1 (wild-type or Y433F). In these cells, MST1 remained inactive as reflected by unchanged pMST1/2 and pMOB1, except if okadaic acid, known to enhance pMST1/2 by PP2A phosphatase inhibition [56], was added (Fig. S4C). This treatment dramatically increased pMOB 1 along with pMST1/2 detection as a doublet in mock and MST1-overexpressing cells (Fig. 6b, S4C). Notably, FGFR4 suppressed pMST1/2 in okadaic acid-treated mock and wild-type MST1 cells, whereas pMST1/2 and pMOB1 were increased after MST1Y433F and FGFR4 co-expression (Fig. 6b, S4C).

In these okadaic acid-treated cells, MST1 or MST2 knockdown suppressed pMOB1, MST2 depletion being most 

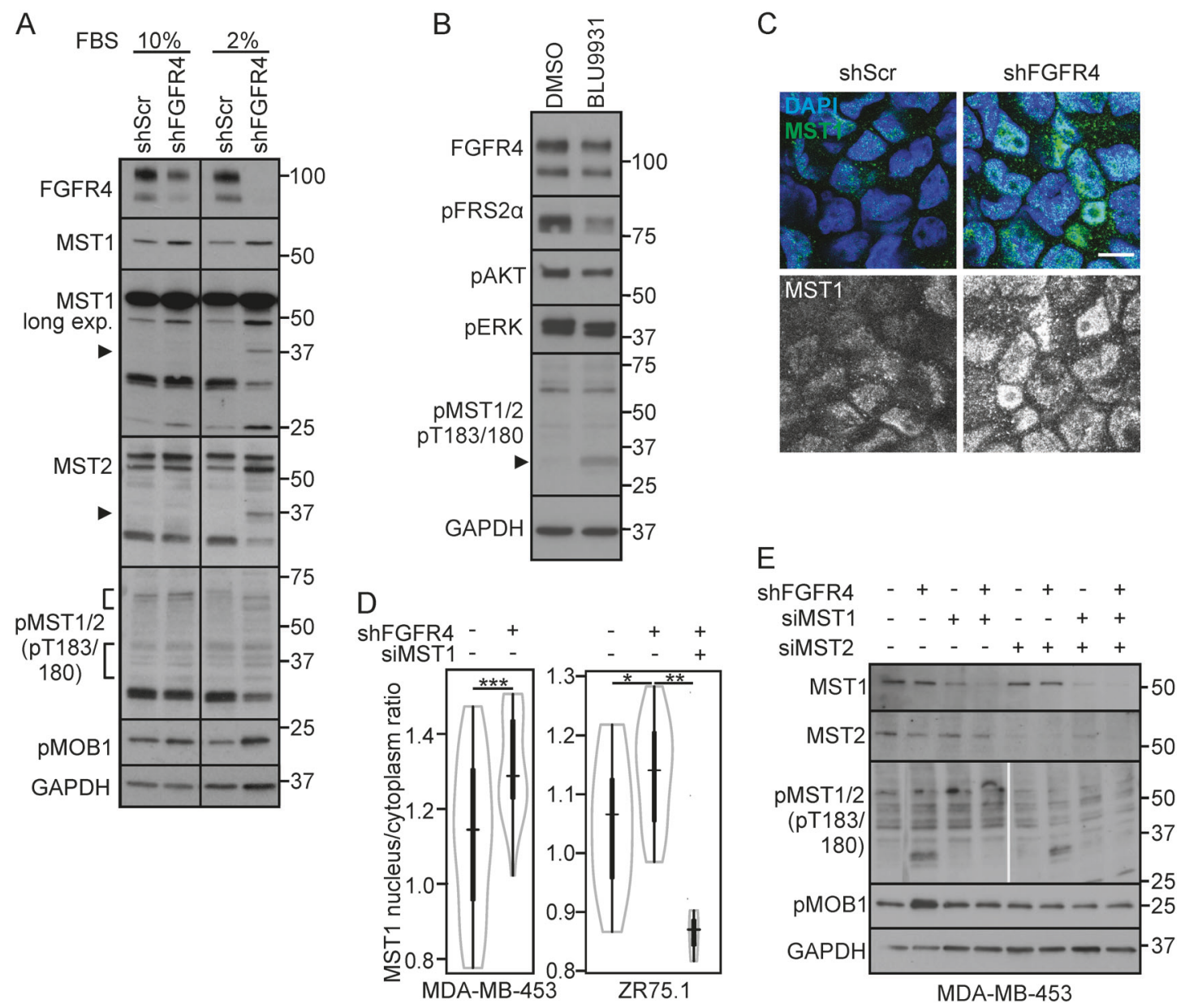

Fig. 5 FGFR4 suppresses MST1/2 activation and nuclear localization in cancer cell spheres. a shScr and shFGFR4 MDA-MB-453 cell spheres were cultured under non-adherent conditions $(10 \%$ or $2 \%$ FBS), and subjected to immunoblotting. Arrowhead; cleaved N-terminal MST1/2 (in 2\% FBS), brackets highlight the fragments of autoactivated MST1/2. b MDA-MB-453 cell spheres were treated with 100 nм BLU9931 for 15 min, and subjected to immunoblotting. c, d shScr and shFGFR4 MDA-MB-453 and ZR-75.1 spheres were

analyzed for MST1 expression by $\mathbf{c}$ immunofluorescence, and d MST1 nuclear/cytoplasmic ratio was quantified $(n=4-6$ MDA-MB-453 spheres, $\geq 6$ microscopic fields/sphere; $n=2-3$ ZR-75.1 spheres, $\geq 8$ microscopic fields/ sphere; mean \pm SEM of two independent experiments. Scale bar $10 \mu \mathrm{m}$. e shScr and shFGFR4 MDA-MB-453 cells were transfected with indicated siRNAs before sphere formation, cultured under non-adherent conditions (1\% FBS) for $48 \mathrm{~h}$, and subjected to immunoblotting

effective, and decreasing also pMST1/2 doublet (Fig. 6c). Strikingly, MST2 knockdown also blocked the MST1Y433F-mediated pMST1/2 induction (Fig. 6c). After MST2 depletion, MST1-Y433F even reduced pMOB1, which effect was reverted by FGFR4 (Fig. 6c). Considering such MST2dependence of FGFR4-mediated MST1 regulation, suggestive of key changes in MST1/2 heterodimer interactions and activity, we tested if FGFR4 can directly alter MST1 activity. The recombinant MST1 activity, measured as ADP generation in vitro, was not altered by recombinant FGFR4, while being inhibited by MST1 inhibitor XMU-MP-1 [30] (Fig. S5A, B). Altogether, this is consistent with mutually competitive MST2 cytoplasmic functions reflected by pMOB1, and pro-apoptotic MST1/2 heterotypic activation, whereby specifically the MST1/2 activation is suppressed by FGFR4dependent MST1-Y433 phosphorylation.

\section{Regulation of YAP differs from pro-apoptotic MST1/ 2 activation}

We next analyzed protein alterations and target gene transcription of the canonical Hippo pathway effector YAP, to test if FGFR4 affects cytoplasmic MST1/2 signaling [18, 19]. The nuclear/cytoplasmic YAP ratio remained essentially unaltered in 3D cell spheres (Fig. S6A). However, YAP localization shifted from irregular to more polarized and membrane-proximal pattern after FGFR4 silencing in MDA-MB-453 (Fig. S6B). This coincided with subtle alterations in inactive ( $\mathrm{pS} 127$ YAP), total, or active (non-pS127) YAP (Fig. S6C, D), without significant changes in mRNAs for the canonical YAP target genes CTGF, CYR61, and ANKRD1 (Fig. S6E). In ZR-75.1, FGFR4 depletion enhanced inactive/ 


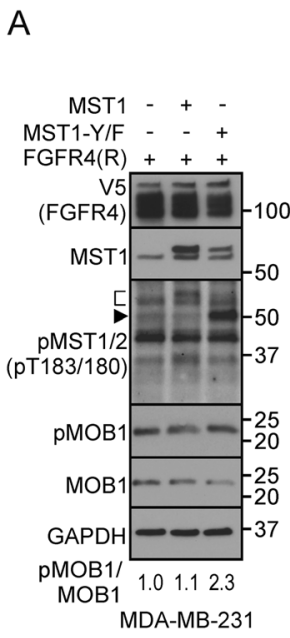

B

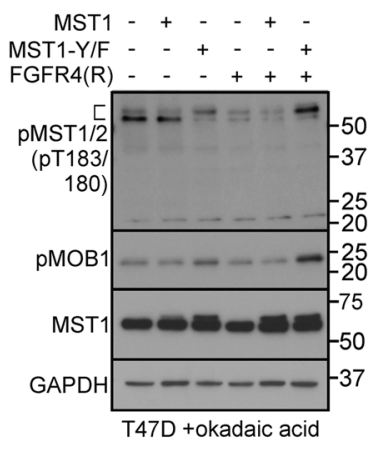

C

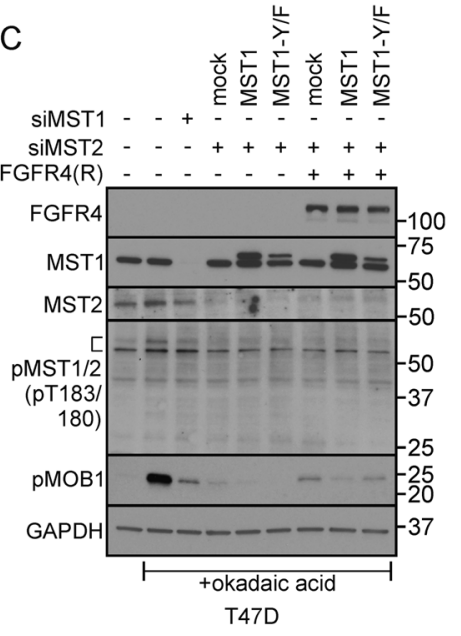

Fig. 6 MST1-Y433F phosphosite mutant restores MST1/2 activation in FGFR4 expressing cancer cells. a MDA-MB-231 cells cotransfected with FGFR4 (R) and wild-type or phosphosite mutant MST1-Y433F were subjected to immunoblotting as indicated. Ratio of pMOB1/MOB1 is indicated below the immunoblot panel. b T47D cells (co-)transfected with wild-type or MST1-Y433F alone or with FGFR4 (R) were treated with $1 \mu \mathrm{m}$ okadaic acid for $1 \mathrm{~h}$ before cell

total and decreased active YAP in more MST2-dependent manner (Fig. S7A). Therefore, FGFR4 had variable effects on YAP, which did not concur with the proapoptotic $\mathrm{pMST} 1 / 2$ regulation.

\section{Apoptosis evasion by FGFR4 encompasses co- targetable vulnerabilities with mitochondrial apoptosis pathway and HER2/EGFR, AKT, and mTORC1 signaling axes}

To consider the overall impact of FGFR4 in human breast cancer, we systematically analyzed (phospho)protein alterations in FGFR4-overexpressing human tumors using TCGA reverse phase protein array (RPPA) data. Significantly, the pro-apoptotic protein BAX, DNA repair protein RAD50, and YAP (pS127 and total) were downregulated, along with the strongest decrease in progesterone receptor, in FGFR4-overexpressing tumors (Fig. 7a). Moreover, FGFR4 overexpression correlated with increased total and pY1248-HER2/ERBB2, pY1068-EGFR, and pY1248-ERBB3, in conjunction with adverse patient outcome (Fig. 7a, S7B; TCGA [4]).

To relate these alterations in human tumors to the FGFR4-dependent apoptosis evasion, we further analyzed how FGFR4 regulates the cell phenotype in 3D matrixembedded cultures. The actively growing Ki67-positive FGFR4 $^{+}$MDA-MB-453 cells responded to the specific FGFR4 inhibitor BLU9931 treatment (100 nM) with a tendency to reduced proliferation as assessed by Ki67positivity (Fig. $7 \mathrm{~b}, \mathrm{c} ; P=0.07$ ). More strikingly, pro- lysis, and subjected to immunoblotting. See corresponding T47D immunoblots without okadaic acid in Fig. S4C. c T47D cells with indicated siRNAs, and (co-)transfected with wild-type or MST1Y433F alone or with FGFR4 (R) were treated with $1 \mu \mathrm{M}$ okadaic acid as above, and subjected to immunoblotting. a-c Brackets and arrowhead indicate the activated pMST1/2 fragments. $N=2$ independent repeats

apoptotic BAX protein positivity coupled to apoptotic membrane blebbing increased significantly (Fig. 7b, d; $P=$ 0.001). Untreated ZR-75.1 cells instead grew poorly, but showed prominent Ki67-positive growth upon FGF1 treatment, which was inhibited by BLU9931 (Fig. 7e, f; $P=$ 0.01), whereas here the concomitant increase in BAX did not reach significance (Fig. 7e, g).

Considering these indications, further linking FGFR4 to apoptosis evasion in human tumors and 3D cultures, we next systematically interrogated the association of FGFR4 activity with drug sensitivity and resistance, targeting clinically actionable vulnerabilities of cancer cells. To this end, we screened a comprehensive oncology library comprised of 527 approved and investigational drugs in combination with BLU9931 in MDA-MB-453 cells. Consistent with known signaling pathway crosstalk [57-59], these cells were relatively sensitive to several (pan)-PI3K/mTOR and AKT inhibitors, and to a few histone deacetylase (HDAC) inhibitors, as assessed by DSS [36] (Table S2). Moreover, MDA-MB-453 showed distinctive sensitivity towards BET/bromodomain inhibitors, which as with most drugs, was not further affected by BLU9931 at the used concentration (100 nM; Table S2).

Markedly, combination treatment with BLU9931 in comprehensive drug testing sensitized the FGFR4 inhibitor-treated cells to the modulators of mitochondrial apoptosis pathway, BCL- $\mathrm{X}_{\mathrm{L}}$ inhibitor (A-1155463) and a SMAC mimetic/IAP inhibitor (Birinapant) (Fig. 7h, Table S3). HER2/EGFR, AKT, and mTORC1 inhibitors (rapalogs) likewise showed better efficacy in BLU9931 treated cells, and importantly, compounds with same mechanism of action showed 

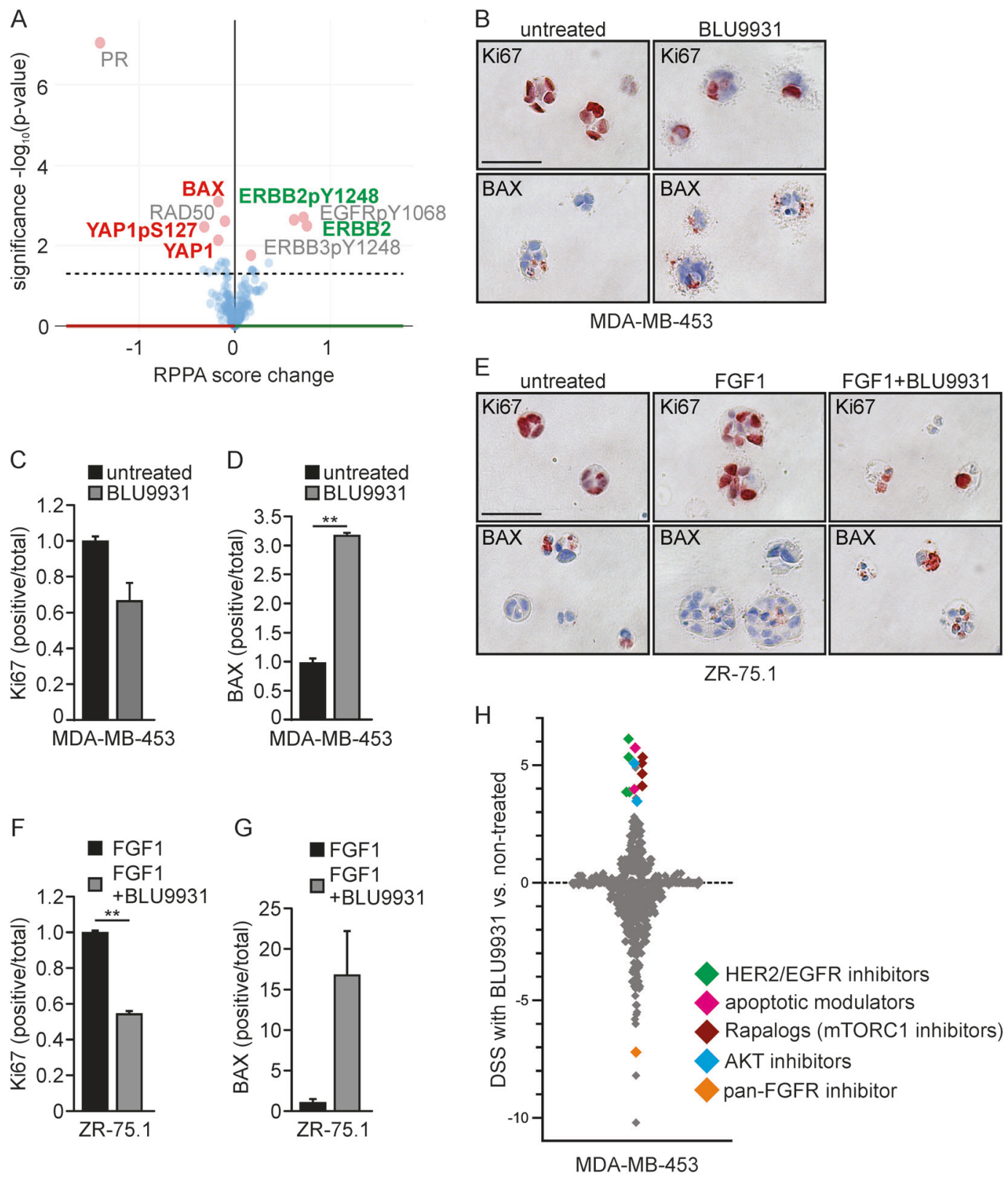

Fig. 7 FGFR4 confers resistance to apoptotic modulators in comprehensive drug screen. a (Phospho)protein changes in TCGA RPPA data [4] associated with FGFR4 upregulation in breast cancer, visualized using cBioPortal (RPPA score change in breast cancer tumors with and without alterations in FGFR4; (mean FGFR4 altered - mean FGFR4 unaltered) [46, 47]. The most significantly up- and downregulated proteins are highlighted (pink dots); ERBB2, alternative name of HER2; PR, progesterone receptor. b-g Fibrin embedded single-cell suspensions of $\mathbf{b}-\mathbf{d}$ MDA-MB-453 and $\mathbf{e}-\mathbf{g}$ ZR-75.1 cells were treated with $100 \mathrm{~nm}$ BLU9931 and/or $30 \mathrm{ng} / \mathrm{ml}$ FGF1 over a 13-14-day culture, fixed, embedded into paraffin for sectioning, and subjected to immunohistochemistry for Ki67 and BAX expression. Positively

comparable shift in DSSs (Fig. 7h, Table S3). Moreover, MDA-MB-453 cells were sensitive to pan-FGFR inhibitor (LY-2874455), whereas the cells treated with BLU9931 did not respond to additional FGFR targeting over a broad concentration range (Fig. 7h, Table S3).

stained vs. total number of cells per colony were counted $(N=30$, mean $\pm \mathrm{SD}, * * P<0.01)$. Scale bar $50 \mu \mathrm{m}$ in $\mathbf{b}$ and $\mathbf{e}$. b For comprehensive drug sensitivity testing $(N=1)$, MDA-MB-453 cells were treated with 527 compounds in five-point dose either alone or in combination with specific FGFR4 inhibitor BLU9931. Dotplot showing the difference in DSS (drug sensitivity score) for cells in treatment combination with BLU9931 (100 nM) versus single agent treatments. Negative values are compounds inducing larger decreases in viability as single agents; positive scores indicate compounds yielding larger decreases in viability in the presence of BLU9931. Colors demarcate compounds with similar class

\section{Discussion}

Apoptosis evasion is one of the classical hallmarks of cancer. Here, we identified the apoptosis-promoting MST1/2 as unique FGFR4 substrates by an unbiased 
in vitro screen. We provide evidence suggesting that by Y433-MST1 phosphorylation, FGFR4 inhibits MST1/2 activation. In endogenous $\mathrm{FGFR} 4^{+} / \mathrm{HER} 2^{+}$breast cancer cell model, this inhibition was essential to counteract apoptosis induction. By this mechanism, FGFR4 can increase cell survival in breast cancers, where fast proliferation is driven by HER/ERBB and FGFR4 signaling [4, 5]. In addition, supporting this conclusion, FGFR4 overexpression was associated with poor HER $2^{+}$breast cancer patient survival.

MST1 and MST2 are the core kinases of mammalian Hippo tumor suppressor pathway, which complex and context-dependent regulatory mechanisms in health and disease still remain unclear. What is known is that MST1/2 are activated via homo- or heterodimerization followed by trans-phosphorylation at T183/180 [19, 60]. Upon apoptosis, caspase-3-mediated cleavage removes the C-terminal MST1/2 regulatory and nuclear export signals, triggering nuclear translocation of the active N-terminal kinase [20, 21]. Although MST1/2 activity has been implicated in histone phosphorylation and chromatin condensation, functional contribution of endogenous MST1/2 to apoptosis, downstream of caspase-3, remains unclear $[19,21,61$, 62]. Here, we show that in FGFR $4^{+} / \mathrm{HER} 2^{+}$breast cancer cells, particularly under tumor-mimicking 3D conditions, FGFR4 depletion-induced MST1/2 autophosphorylation coupled with MST1 cleavage and nuclear localization without additional stimuli. This resulted in significant apoptosis induction, which was rescued back to low levels by double knockdown of FGFR4 with MST1 or MST2. In cells with endogenous FGFR4, however, MST1 or MST2 silencing did not reduce apoptosis, suggesting that the cells have developed dependency of the herein identified FGFR4-mediated suppression of apoptosis. Moreover, pharmacological FGFR4 targeting specifically sensitized the cells, in addition to HER2/EGFR and PI3K/AKT/ mTOR inhibitors, to the modulators of mitochondrial apoptosis pathway, suggesting interesting possibilities also for combinatorial treatments to effectively halt the FGFR4 ${ }^{+} /$HER2 ${ }^{+}$cancers [63].

Previously, other kinases have been indicated in positive and negative MST1/2 regulation [31, 64-67]. In neuronal cells, MST1 phosphorylation by non-receptor tyrosine kinase c-Abl at Y433 (conserved site in mammals, absent in MST2) leads to MST1 stabilization and activation thus increasing cell death [68], an opposite outcome from the herein identified FGFR4-mediated inhibition of MST1/2 and apoptosis. Moreover, c-Abl-mediated phosphorylation of Y81 on MST2 (species-conserved site, absent in MST1) enhances MST2 activation [69]. Our current results indicate that, although involving specific MST1/N cleavage and suppression upon MST1 phosphorylation (pY433), the apoptosis induction by FGFR4 depletion required combined activity of MST1 and MST2, whereas these individual kinases were regulated even in an opposite manner. This can be related to the mechanisms whereby AKT signaling differentially regulates MST1 and MST2 [54, 70], and MST1/2 integrate signals from Ras signaling (Raf-1, RASSF1A) [54, 60, 71, 72]. As recombinant MST1 activity was not inhibited by FGFR4 in vitro, we suggest that FGFR4 likely alters MST1/2 activities via MST1-pY433regulated interactions. Further context-dependent aspects of MST2 phosphorylation, (hetero)dimerization, signaling scaffolds, and activity or cleavage regulation will remain interesting future study subjects. Moreover, the other herein identified unique MST1-pS410 phosphosite detected only in the absence of FGFR4 activity and pY433, raises the possibility of mutually regulated phosphorylation of these residues. The two MST1 phosphosites detected independently of pY433 have been characterized; S320 as a phosphorylation site for anti-apoptotic CK2 [73], and T177 as a MST1 activity-dependent site [74]. Therefore, the contextdependent net effects of all MST1 phosphosites on its activity regulation, including contributions by kinases other than FGFR4, will be of interest. Nevertheless, current results show that active FGFR4 tyrosine phosphorylates and inactivates the pro-apoptotic MST1/2 serine/threonine kinase in breast cancer cells, thus revealing a novel mechanism of RTK-mediated apoptosis evasion and oncogenic FGFR4 function.

Apart from pro-apoptotic nuclear functions, cytoplasmic MST1/2 act on canonical Hippo signaling, which negatively regulates YAP by serine phosphorylation and cytoplasmic retention [19, 75]. In cancer, however, non-canonical regulation of YAP expression, localization and activity frequently prevails along with the loss of balancing feed-back mechanisms of normal cellular homeostasis [18, 75, 76]. We tested if FGFR4-mediated pro-apoptotic MST1/2 inhibition also regulated YAP. Although YAP (pS127 and total) was decreased in FGFR4-overexpressing breast cancers (TCGA RPPA), the FGFR4-dependent and -independent modulation of MST1/2 had variable effects on YAP S127 phosphorylation or localization. This is consistent with accumulating evidence acknowledging cell polarity, junctional complexes, and cytoskeletal signals as important YAP regulators even independent of MST1/2 [17, 66]. A further interesting aspect is that FGFRl, -2 , and -4 are transcriptional targets of YAP, and a feed-forward loop has been described between YAP and FGFR signaling in cholangiocarcinoma and ovarian cancer [77, 78].

In conclusion, the identified oncogenic FGFR4 activity explains mechanistically how RTKs such as FGFR4 can confer aggressiveness to certain cancer cells via apoptosis resistance. As FGFR4 is suitable for targeting, these results raise tempting questions, whether FGFR4 inhibition in $\mathrm{HER}^{+}{ }^{+}$breast cancer would release the intrinsic MST1/2- 
mediated apoptotic machinery. And if so, would it offer increased efficacy in combination with targeting HER2, AKT/mTOR, and/or mitochondrial apoptosis pathways in this cancer subset, or in other FGFR4-overexpressing cancers? Nonetheless, the identification of MST1/2 as direct substrates for FGFR4, and FGFR4-dependent inhibition of MST1/2-dependent apoptosis, highlights interesting avenues for FGFR4 targeting in anticancer treatments.

Acknowledgements We thank Anastasiya Chernenko for excellent technical assistance, Dr. Jin Cheng (Motiff Cancer Center, Florida, USA) for Flag-tagged MST1 and MST2 plasmids, Julia Casado for initial TCGA expression analysis, and Biomedicum Imaging Unit, University of Helsinki for imaging facilities. This work was funded by the Karolinska Institutet, KI Strategic Research Program in Cancer (StratCan-KICancer), Swedish Cancer Society (Cancerfonden), Swedish Research Council (Vetenskapsrådet), the University of Helsinki, Academy of Finland, Sigrid Jusélius Foundation, Knut and Alice Wallenberg Foundation, Science for Life Laboratory, Magnus Ehrnrooth Foundation, Medicinska understödsföreningen Liv och hälsa, and the The Finnish Society of Sciences and Letters.

\section{Compliance with ethical standards}

Conflict of interest The authors declare that they have no conflict of interest.

Publisher's note: Springer Nature remains neutral with regard to jurisdictional claims in published maps and institutional affiliations.

Open Access This article is licensed under a Creative Commons Attribution 4.0 International License, which permits use, sharing, adaptation, distribution and reproduction in any medium or format, as long as you give appropriate credit to the original author(s) and the source, provide a link to the Creative Commons license, and indicate if changes were made. The images or other third party material in this article are included in the article's Creative Commons license, unless indicated otherwise in a credit line to the material. If material is not included in the article's Creative Commons license and your intended use is not permitted by statutory regulation or exceeds the permitted use, you will need to obtain permission directly from the copyright holder. To view a copy of this license, visit http://creativecommons. org/licenses/by/4.0/

\section{References}

1. Lemmon MA, Schlessinger J. Cell signaling by receptor tyrosine kinases. Cell. 2010;141:1117-34.

2. Babina IS, Turner NC. Advances and challenges in targeting FGFR signalling in cancer. Nat Rev Cancer. 2017;17:318-32.

3. Weinstein M, Xu X, Ohyama K, Deng CX. FGFR-3 and FGFR-4 function cooperatively to direct alveogenesis in the murine lung. Development. 1998;125:3615-23.

4. Cancer Genome Atlas Network. Comprehensive molecular portraits of human breast tumours. Nature. 2012;490:61-70.

5. Kothari V, Wei I, Shankar S, Kalyana-Sundaram S, Wang L, Ma LW, et al. Outlier kinase expression by RNA sequencing as targets for precision therapy. Cancer Discov. 2013;3:280-93.

6. Tucker JA, Klein T, Breed J, Breeze AL, Overman R, Phillips C, et al. Structural insights into FGFR kinase isoform selectivity: diverse binding modes of AZD4547 and ponatinib in complex with FGFR1 and FGFR4. Structure. 2014;22:1764-74.
7. Lesca E, Lammens A, Huber R, Augustin M. Structural analysis of the human fibroblast growth factor receptor 4 kinase. J Mol Biol. 2014:426:3744-56.

8. Huang Z, Tan L, Wang H, Liu Y, Blais S, Deng J, et al. DFG-out mode of inhibition by an irreversible type-1 inhibitor capable of overcoming gate-keeper mutations in FGF receptors. ACS Chem Biol. 2015;10:299-309.

9. Mo C, Zhang Z, Guise CP, Li X, Luo J, Tu Z, et al. 2aminopyrimidine derivatives as new selective fibroblast growth factor receptor 4 (FGFR4) inhibitors. ACS Med Chem Lett. 2017;8:543-8

10. Bray F, Ferlay J, Soerjomataram I, Siegel RL, Torre LA, Jemal A. Global cancer statistics 2018: GLOBOCAN estimates of incidence and mortality worldwide for 36 cancers in 185 countries. CA Cancer J Clin. 2018;68:394-424.

11. Pfeffer CM, Singh ATK. Apoptosis: a target for anticancer therapy. Int J Mol Sci. 2018;19:pii: E448.

12. Bange J, Prechtl D, Cheburkin Y, Specht K, Harbeck N, Schmitt M, et al. Cancer progression and tumor cell motility are associated with the FGFR4 $\operatorname{Arg}(388)$ allele. Cancer Res. 2002; 62:840-7.

13. Sugiyama N, Varjosalo M, Meller P, Lohi J, Hyytiäinen M, Kilpinen S, et al. Fibroblast growth factor receptor 4 regulates tumor invasion by coupling fibroblast growth factor signaling to extracellular matrix degradation. Cancer Res. 2010;70:7851-61.

14. Zaid TM, Yeung TL, Thompson MS, Leung CS, Harding T, Co $\mathrm{NN}$, et al. Identification of FGFR4 as a potential therapeutic target for advanced-stage, high-grade serous ovarian cancer. Clin Cancer Res. 2013;19:809-20.

15. Roidl A, Berger HJ, Kumar S, Bange J, Knyazev P, Ullrich A. Resistance to chemotherapy is associated with fibroblast growth factor receptor 4 up-regulation. Clin Cancer Res. 2009;15:205866.

16. Priedigkeit N, Hartmaier RJ, Chen Y, Vareslija D, Basudan A, Watters RJ, et al. Intrinsic subtype switching and acquired ERBB2/HER2 amplifications and mutations in breast cancer brain metastases. JAMA Oncol. 2017;3:666-71.

17. Hauri S, Wepf A, van Drogen A, Varjosalo M, Tapon N, Aebersold $\mathrm{R}$, et al. Interaction proteome of human Hippo signaling: modular control of the co-activator YAP1. Mol Syst Biol. 2013;9:713.

18. Johnson R, Halder G. The two faces of Hippo: targeting the Hippo pathway for regenerative medicine and cancer treatment. Nat Rev Drug Discov. 2014;13:63-79.

19. Galan JA, Avruch J. MST1/MST2 protein kinases: regulation and physiologic roles. Biochemistry. 2016;55:5507-19.

20. Graves JD, Gotoh Y, Draves KE, Ambrose D, Han DK, Wright $\mathrm{M}$, et al. Caspase-mediated activation and induction of apoptosis by the mammalian Ste20-like kinase Mst1. EMBO J. 1998; 17:2224-34

21. Ura S, Masuyama N, Graves JD, Gotoh Y. Caspase cleavage of MST1 promotes nuclear translocation and chromatin condensation. Proc Natl Acad Sci USA. 2001;98:10148-53.

22. Graves JD, Draves KE, Gotoh Y, Krebs EG, Clark EA. Both phosphorylation and caspase-mediated cleavage contribute to regulation of the Ste20-like protein kinase Mst1 during CD95/Fasinduced apoptosis. J Biol Chem. 2001;276:14909-15.

23. Lee KK, Ohyama T, Yajima N, Tsubuki S, Yonehara S. MST, a physiological caspase substrate, highly sensitizes apoptosis both upstream and downstream of caspase activation. J Biol Chem. 2001;276:19276-85.

24. Seidel C, Schagdarsurengin U, Blumke K, Wurl P, Pfeifer GP, Hauptmann S, et al. Frequent hypermethylation of MST1 and MST2 in soft tissue sarcoma. Mol Carcinog. 2007;46:865-71.

25. Zhou D, Conrad C, Xia F, Park JS, Payer B, Yin Y, et al. Mst1 and Mst2 maintain hepatocyte quiescence and suppress 
hepatocellular carcinoma development through inactivation of the Yap1 oncogene. Cancer Cell. 2009;16:425-38.

26. Lu L, Li Y, Kim SM, Bossuyt W, Liu P, Qiu Q, et al. Hippo signaling is a potent in vivo growth and tumor suppressor pathway in the mammalian liver. Proc Natl Acad Sci USA. 2010;107:1437-42.

27. Song H, Mak KK, Topol L, Yun K, Hu J, Garrett L, et al. Mammalian Mst1 and Mst2 kinases play essential roles in organ size control and tumor suppression. Proc Natl Acad Sci USA. 2010;107:1431-6.

28. Ethier SP, Mahacek ML, Gullick WJ, Frank TS, Weber BL. Differential isolation of normal luminal mammary epithelial cells and breast cancer cells from primary and metastatic sites using selective media. Cancer Res. 1993;53:627-35.

29. Hagel M, Miduturu C, Sheets M, Rubin N, Weng W, Stransky N, et al. First selective small molecule inhibitor of FGFR4 for the treatment of hepatocellular carcinomas with an activated FGFR4 signaling pathway. Cancer Discov. 2015;5:424-37.

30. Fan F, He Z, Kong LL, Chen Q, Yuan Q, Zhang S, et al. Pharmacological targeting of kinases MST1 and MST2 augments tissue repair and regeneration. Sci Transl Med. 2016;8:352ra108.

31. Bi W, Xiao L, Jia Y, Wu J, Xie Q, Ren J, et al. c-Jun N-terminal kinase enhances MST1-mediated pro-apoptotic signaling through phosphorylation at serine 82. J Biol Chem. 2010;285: 6259-64.

32. Varjosalo M, Björklund M, Cheng F, Syvanen H, Kivioja T, Kilpinen S, et al. Application of active and kinase-deficient kinome collection for identification of kinases regulating hedgehog signaling. Cell. 2008;133:537-48.

33. Sugiyama N, Varjosalo M, Meller P, Lohi J, Chan KM, Zhou Z, et al. FGF receptor-4 (FGFR4) polymorphism acts as an activity switch of a membrane type 1 matrix metalloproteinase-FGFR4 complex. Proc Natl Acad Sci USA. 2010;107:15786-91.

34. Tatti O, Vehviläinen P, Lehti K, Keski-Oja J. MT1-MMP releases latent TGF-betal from endothelial cell extracellular matrix via proteolytic processing of LTBP-1. Exp Cell Res. 2008;314: 2501-14.

35. von Nandelstadh P, Grönholm M, Moza M, Lamberg A, Savilahti $\mathrm{H}$, Carpen O. Actin-organising properties of the muscular dystrophy protein myotilin. Exp Cell Res. 2005;310:131-9.

36. von Nandelstadh P, Gucciardo E, Lohi J, Li R, Sugiyama N, Carpen $\mathrm{O}$, et al. Actin-associated protein palladin promotes tumor cell invasion by linking extracellular matrix degradation to cell cytoskeleton. Mol Biol Cell. 2014;25:2556-70.

37. Lehti K, Lohi J, Juntunen MM, Pei D, Keski-Oja J. Oligomerization through hemopexin and cytoplasmic domains regulates the activity and turnover of membrane-type 1 matrix metalloproteinase. J Biol Chem. 2002;277:8440-8.

38. Zhou H, Ye M, Dong J, Corradini E, Cristobal A, Heck AJ, et al. Robust phosphoproteome enrichment using monodisperse microsphere-based immobilized titanium (IV) ion affinity chromatography. Nat Protoc. 2013;8:461-80.

39. Taus T, Kocher T, Pichler P, Paschke C, Schmidt A, Henrich C, et al. Universal and confident phosphorylation site localization using phosphoRS. J Proteome Res. 2011;10:5354-62.

40. Wang C, Turunen SP, Kummu O, Veneskoski M, Lehtimäki J, Nissinen AE, et al. Natural antibodies of newborns recognize oxidative stress-related malondialdehyde acetaldehyde adducts on apoptotic cells and atherosclerotic plaques. Int Immunol. 2013;25:575-87.

41. Yadav B, Pemovska T, Szwajda A, Kulesskiy E, Kontro M, Karjalainen R, et al. Quantitative scoring of differential drug sensitivity for individually optimized anticancer therapies. Sci Rep. 2014;4:5193.

42. Tatti O, Arjama M, Ranki A, Weiss SJ, Keski-Oja J, Lehti K. Membrane-type-3 matrix metalloproteinase (MT3-MMP) functions as a matrix composition-dependent effector of melanoma cell invasion. PLoS ONE. 2011;6:e28325.

43. Tatti O, Gucciardo E, Pekkonen P, Holopainen T, Louhimo R, Repo P, et al. MMP16 mediates a proteolytic switch to promote cell-cell adhesion, collagen alignment, and lymphatic invasion in melanoma. Cancer Res. 2015;75:2083-94.

44. Rantanen V, Valori M, Hautaniemi S. Anima: modular workflow system for comprehensive image data analysis. Front Bioeng Biotechnol. 2014;2:25.

45. Zhao B, Li L, Tumaneng K, Wang CY, Guan KL. A coordinated phosphorylation by Lats and CK1 regulates YAP stability through SCF(beta-TRCP). Genes Dev. 2010;24:72-85.

46. Cerami E, Gao J, Dogrusoz U, Gross BE, Sumer SO, Aksoy BA, et al. The cBio cancer genomics portal: an open platform for exploring multidimensional cancer genomics data. Cancer Discov. 2012;2:401-4.

47. Gao J, Aksoy BA, Dogrusoz U, Dresdner G, Gross B, Sumer SO, et al. Integrative analysis of complex cancer genomics and clinical profiles using the cBioPortal. Sci Signal. 2013;6:pl1.

48. Parker JS, Mullins M, Cheang MC, Leung S, Voduc D, Vickery $\mathrm{T}$, et al. Supervised risk predictor of breast cancer based on intrinsic subtypes. J Clin Oncol. 2009;27:1160-7.

49. Holliday DL, Speirs V. Choosing the right cell line for breast cancer research. Breast Cancer Res. 2011;13:215.

50. Ruhe JE, Streit S, Hart S, Wong CH, Specht K, Knyazev P, et al. Genetic alterations in the tyrosine kinase transcriptome of human cancer cell lines. Cancer Res. 2007;67:11368-76.

51. Roidl A, Foo P, Wong W, Mann C, Bechtold S, Berger HJ, et al. The FGFR4 Y367C mutant is a dominant oncogene in MDAMB453 breast cancer cells. Oncogene. 2010;29:1543-52.

52. Ulaganathan VK, Sperl B, Rapp UR, Ullrich A. Germline variant FGFR4p.G388R exposes a membrane-proximal STAT3 binding site. Nature. 2015;528:570-4.

53. Praskova M, Xia F, Avruch J. MOBKL1A/MOBKL1B phosphorylation by MST1 and MST2 inhibits cell proliferation. Curr Biol. 2008;18:311-21.

54. Romano D, Matallanas D, Weitsman G, Preisinger C, Ng T, Kolch W. Proapoptotic kinase MST2 coordinates signaling crosstalk between RASSF1A, Raf-1, and Akt. Cancer Res. 2010;70:1195-203.

55. Dupont S, Morsut L, Aragona M, Enzo E, Giulitti S, Cordenonsi $\mathrm{M}$, et al. Role of YAP/TAZ in mechanotransduction. Nature. 2011;474:179-83.

56. Hata Y, Timalsina S, Maimaiti S. Okadaic acid: a tool to study the hippo pathway. Mar Drugs. 2013;11:896-902.

57. Tiong KH, Tan BS, Choo HL, Chung FF, Hii LW, Tan SH, et al. Fibroblast growth factor receptor 4 (FGFR4) and fibroblast growth factor 19 (FGF19) autocrine enhance breast cancer cells survival. Oncotarget. 2016;7:57633-50.

58. McKinnon T, Venier R, Yohe M, Sindiri S, Gryder BE, Shern JF, et al. Functional screening of FGFR4-driven tumorigenesis identifies $\mathrm{PI} 3 \mathrm{~K} / \mathrm{mTOR}$ inhibition as a therapeutic strategy in rhabdomyosarcoma. Oncogene. 2018;37:2630-44.

59. Katoh M. Fibroblast growth factor receptors as treatment targets in clinical oncology. Nat Rev Clin Oncol. 2019;16:105-22.

60. Praskova M, Khoklatchev A, Ortiz-Vega S, Avruch J. Regulation of the MST1 kinase by autophosphorylation, by the growth inhibitory proteins, RASSF1 and NORE1, and by Ras. Biochem J. 2004;381:453-62.

61. Cheung WL, Ajiro K, Samejima K, Kloc M, Cheung P, Mizzen $\mathrm{CA}$, et al. Apoptotic phosphorylation of histone $\mathrm{H} 2 \mathrm{~B}$ is mediated by mammalian sterile twenty kinase. Cell. 2003;113:507-17.

62. Pefani DE, Tognoli ML, Pirincci Ercan D, Gorgoulis V, O’Neill E. MST2 kinase suppresses rDNA transcription in response to DNA damage by phosphorylating nucleolar histone H2B. EMBO J. 2018; 37:pii: e98760. 
63. Ye T, Wei X, Yin T, Xia Y, Li D, Shao B, et al. Inhibition of FGFR signaling by PD173074 improves antitumor immunity and impairs breast cancer metastasis. Breast Cancer Res Treat. 2014;143:435-46.

64. Boggiano JC, Vanderzalm PJ, Fehon RG. Tao-1 phosphorylates Hippo/MST kinases to regulate the Hippo-Salvador-Warts tumor suppressor pathway. Dev Cell. 2011;21:888-95.

65. Poon CL, Lin JI, Zhang X, Harvey KF. The sterile 20-like kinase Tao-1 controls tissue growth by regulating the Salvador-WartsHippo pathway. Dev Cell. 2011;21:896-906.

66. Meng Z, Moroishi T, Guan KL. Mechanisms of Hippo pathway regulation. Genes Dev. 2016;30:1-17.

67. Zhao S, Yin J, Zhou L, Yan F, He Q, Huang L, et al. Hippo/ MST1 signaling mediates microglial activation following acute cerebral ischemia-reperfusion injury. Brain Behav Immun. 2016;55:236-48.

68. Xiao L, Chen D, Hu P, Wu J, Liu W, Zhao Y, et al. The c-AblMST1 signaling pathway mediates oxidative stress-induced neuronal cell death. J Neurosci. 2011;31:9611-9.

69. Liu W, Wu J, Xiao L, Bai Y, Qu A, Zheng Z, et al. Regulation of neuronal cell death by c-Abl-Hippo/MST2 signaling pathway. PLoS ONE. 2012;7:e36562.

70. Jang SW, Yang SJ, Srinivasan S, Ye K. Akt phosphorylates MstI and prevents its proteolytic activation, blocking FOXO3 phosphorylation and nuclear translocation. J Biol Chem. 2007;282:30836-44.
71. O'Neill E, Kolch W. Taming the Hippo: Raf-1 controls apoptosis by suppressing MST2/Hippo. Cell Cycle. 2005;4:365-7.

72. Guo C, Zhang X, Pfeifer GP. The tumor suppressor RASSF1A prevents dephosphorylation of the mammalian STE20-like kinases MST1 and MST2. J Biol Chem. 2011;286:6253-61.

73. Servas C, Kiehlmeier S, Hach J, Gross R, Gotz C, Montenarh M. The mammalian STE20-like kinase 1 (MST1) is a substrate for the apoptosis inhibiting protein kinase CK2. Cell Signal. 2017;36: $163-75$.

74. Glantschnig H, Rodan GA, Reszka AA. Mapping of MST1 kinase sites of phosphorylation. Activation and autophosphorylation. J Biol Chem. 2002;277:42987-96.

75. Zanconato F, Cordenonsi M, Piccolo S. YAP/TAZ at the roots of cancer. Cancer Cell. 2016;29:783-803.

76. Chen Q, Zhang N, Xie R, Wang W, Cai J, Choi KS, et al. Homeostatic control of Hippo signaling activity revealed by an endogenous activating mutation in YAP. Genes Dev. 2015;29: 1285-97.

77. Rizvi S, Yamada D, Hirsova P, Bronk SF, Werneburg NW, Krishnan A, et al. A hippo and fibroblast growth factor receptor autocrine pathway in cholangiocarcinoma. J Biol Chem. 2016; 291:8031-47.

78. Hua G, Lv X, He C, Remmenga SW, Rodabough KJ, Dong J, et al. YAP induces high-grade serous carcinoma in fallopian tube secretory epithelial cells. Oncogene. 2016;35:2247-65. 